\title{
Workshop on Advanced Computational Materials Science: Application to Fusion and Generation IV Fission Reactors
}

\author{
Sponsored by \\ U.S. Department of Energy \\ Office of Science \\ Office of Nuclear Energy, Science, and Technology \\ Washington, D.C. \\ 31 March - 2 April 2004
}

\author{
Submitted to: \\ Dr. Raymond L. Orbach \\ Director, DOE Office of Science \\ and \\ Dr. William D. Magwood, IV
}

Director, DOE Office of Nuclear Energy, Science, and Technology

on behalf of the workshop participants by the organizers:

Roger E. Stoller, Metals and Ceramics Division

Steven J. Zinkle, Metals and Ceramics Division

Jeffrey A. Nichols, Computer Science and Mathematics Division

William R. Corwin, Metals and Ceramics Division

Oak Ridge National Laboratory

30 June 2004 


\section{Table of Contents}

$\begin{array}{lll}\text { Chapter } & \text { Page }\end{array}$

Executive Summary $\quad 3$

$\begin{array}{lc}\text { Introduction } & 6\end{array}$

1. Session I: $\quad$ Overview of Relevant Irradiation Environments 8

2. Session II: Current State of the Art in Relevant Multiscale Computational Materials $\begin{array}{ll}\text { Science Models } & 16\end{array}$

3. Session III: Goals and Targets for Understanding and Predicting Material Performance 27

4. Session IV: Expected Advances and Contributions of Computational Science 41

Summary and Recommendations $\quad 54$

$\begin{array}{lll}\text { Appendix A } & \text { Workshop Agenda } & 58\end{array}$

$\begin{array}{lll}\text { Appendix B } & \text { Workshop Participants } & 65\end{array}$

$\begin{array}{lll}\text { Appendix C } & \text { Acronyms and Abbreviations } & 67\end{array}$

\section{Acknowledgement}

The ORNL research staff who were responsible for organizing the Workshop and assembling this report gratefully acknowledge the contributions of all the workshop participants to the success of the meeting, and to the technical and editorial content of the report. In particular, those members of the International Advisory Committee who served as session chairmen, Drs. Patrick Ledermann, David Bacon, Nasr Ghoniem, and David Keyes, acted as lead authors for the chapters developed from their respective sessions. 


\section{Executive Summary}

The Workshop on Advanced Computational Materials Science: Application to Fusion and Generation IV Fission Reactors was convened to determine the degree to which an increased effort in modeling and simulation could help bridge the gap between the data that is needed to support the implementation of these advanced nuclear technologies and the data that can be obtained in available experimental facilities.

The need to develop materials capable of performing in the severe operating environments expected in fusion and fission (Generation IV) reactors represents a significant challenge in materials science. There is a range of potential Gen-IV fission reactor design concepts and each concept has its own unique demands. Improved economic performance is a major goal of the Gen-IV designs. As a result, most designs call for significantly higher operating temperatures than the current generation of LWRs to obtain higher thermal efficiency. In many cases, the desired operating temperatures rule out the use of the structural alloys employed today. The very high operating temperature (up to $1000^{\circ} \mathrm{C}$ ) associated with the NGNP is a prime example of an attractive new system that will require the development of new structural materials. Fusion power plants represent an even greater challenge to structural materials development and application. The operating temperatures, neutron exposure levels and thermo-mechanical stresses are comparable to or greater than those for proposed Gen-IV fission reactors. In addition, the transmutation products created in the structural materials by the high energy neutrons produced in the DT plasma can profoundly influence the microstructural evolution and mechanical behavior of these materials.

Although the workshop addressed issues relevant to both Gen-IV and fusion reactor materials, much of the discussion focused on fusion; the same focus is reflected in this report. Most of the physical models and computational methods presented during the workshop apply equally to both types of nuclear energy systems. The primary factor that differentiates the materials development path for the two systems is that nearly prototypical irradiation environments for Gen-IV materials can be found or built in existing fission reactors. This is not the case for fusion. The only fusion-relevant, $14 \mathrm{MeV}$ neutron sources ever built (such as the rotating target neutron sources, RTNS-I and -II at LLNL) were relatively low-power accelerator based systems. The RTNS-II "high" flux irradiation volume was quite small, less than $1 \mathrm{~cm}^{3}$, and only low doses could be achieved. The maximum dose data obtained was much less than 0.1 dpa. Thus, RTNS-II, which last operated in 1986, provided only a limited opportunity for fundamental investigations of the effects of $14 \mathrm{MeV}$ neutrons characteristic of DT fusion.

Historically, both the fusion and fission reactor programs have taken advantage of and built on research carried out by the other program. This leveraging can be expected to continue over the next ten years as both experimental and modeling activities in support of the Gen-IV program grow substantially. The Gen-IV research will augment the fusion studies (and vice versa) in areas where similar materials and exposure conditions are of interest. However, in addition to the concerns that are common to both fusion and advanced fission reactor programs, designers of a future DT fusion reactor have the unique problem of anticipating the effects of the $14 \mathrm{MeV}$ neutron source term. In particular, the question arises whether irradiation data obtained in a near-prototypic irradiation environment such as the IFMIF are needed to verify results obtained from computational materials research. The need for a theory and modeling effort to work hand-in-hand with a complementary experimental program for the purpose of model development and verification, and for validation of model predictions was extensively discussed at the workshop. There was a clear consensus that an IFMIF-like irradiation facility is likely to be required to contribute to this research. However, the question of whether IFMIF itself is needed was explored from two different points of view at the workshop. These complementary (and in some cases opposing) points of view can be coarsely characterized as "scientific" and "engineering."

The recent and anticipated progress in computational materials science presented at the workshop provides some confidence that many of the scientific questions whose answers will underpin 
the successful use of structural materials in a DT fusion reactor can be addressed in a reasonable time frame if sufficient resources are devoted to this effort. For example, advances in computing hardware and software should permit improved (and in some cases the first) descriptions of relevant properties in alloys based on $a b$ initio calculations. Such calculations could provide the basis for realistic interatomic potentials for alloys, including alloy-He potentials, that can be applied in classical molecular dynamics simulations. These potentials must have a more detailed description of many-body interactions than accounted for in the current generation which are generally based on a simple embedding function. In addition, the potentials used under fusion reactor conditions (very high PKA energies) should account for the effects of local electronic excitation and electronic energy loss. The computational cost of using more complex potentials also requires the next generation of massively parallel computers. New results of $a b$ initio and atomistic calculations can be coupled with ongoing advances in kinetic and phase field models to dramatically improve predictions of the non-equilibrium, radiation-induced evolution in alloys with unstable microstructures. This includes phase stability and the effects of helium on each microstructural component.

However, for all its promise, computational materials science is still a house under construction. As such, the current reach of the science is limited. Theory and modeling can be used to develop understanding of known critical physical phenomena, and computer experiments can, and have been used to, identify new phenomena and mechanisms, and to aid in alloy design. However, it is questionable whether the science will be sufficiently mature in the foreseeable future to provide a rigorous scientific basis for predicting critical materials' properties, or for extrapolating well beyond the available validation database.

Two other issues remain even if the scientific questions appear to have been adequately answered. These are licensing and capital investment. Even a high degree of scientific confidence that a given alloy will perform as needed in a particular Gen-IV or fusion environment is not necessarily transferable to the reactor licensing or capital market regimes. The philosophy, codes, and standards employed for reactor licensing are properly conservative with respect to design data requirements. Experience with the U.S. Nuclear Regulatory Commission suggests that only modeling results that are strongly supported by relevant, prototypical data will have an impact on the licensing process. In a similar way, it is expected that investment on the scale required to build a fusion power plant (several billion dollars) could only be obtained if a very high level of confidence existed that the plant would operate long and safely enough to return the investment.

These latter two concerns appear to dictate that an experimental facility capable of generating a sufficient, if limited, body of design data under essentially prototypic conditions (i.e. with $\sim 14 \mathrm{MeV}$ neutrons) will ultimately be required for the commercialization of fusion power. An aggressive theory and modeling effort will reduce the time and experimental investment required to develop the advanced materials that can perform in a DT fusion reactor environment. For example, the quantity of design data may be reduced to that required to confirm model predictions for key materials at critical exposure conditions. This will include some data at a substantial fraction of the anticipated end-of-life dose, which raises the issue of when such an experimental facility is required. Long lead times for construction of complex facilities, coupled with several years irradiation to reach the highest doses, imply that the decision to build any fusion-relevant irradiation facility must be made on the order of 10 years before the design data is needed.

Two related areas of research can be used as reference points for the expressed need to obtain experimental validation of model predictions. Among the lessons learned from ASCI, the importance of code validation and verification was emphasized at the workshop. Despite an extensive investment in theory and modeling of the relevant physics, the NIF is being built at LLNL to verify the performance of the physics codes. Similarly, while the U.S. and international fusion community has invested considerable resources in simulating the behavior of magnetically-confined plasmas, a series of experimental devices (e.g. DIII-D, TFTR, JET, NSTX, and NCSX) have been, or will be, built and 
numerous experiments carried out to validate the predicted plasma performance on the route to ITER and a demonstration fusion power reactor 


\section{Introduction}

A renewed interest in nuclear reactor technology has developed in recent years, in part as a result of international interest in sources of energy that do not produce $\mathrm{CO}_{2}$ as a by-product. One result of this interest was the establishment of the Generation IV International Forum (http:/geniv.ne.doe.gov/ ), which is a group of international governmental entities whose goal is facilitating bilateral and multilateral cooperation related to the development of new nuclear energy systems. At the same time, advances in plasma physics and fusion engineering have raised interest in the feasibility of fusion energy employing the deuterium-tritium (DT) fusion reaction. This is evidenced by the international effort to build the ITER (http://www.ofes.fusion.doe.gov/iter.html) as the first fusion science experiment capable of producing a self-sustaining fusion reaction. ITER is seen as the essential next step on the path of demonstrating the scientific and technological feasibility of fusion energy. Interest in fusion is sufficiently high that international panels have recommended a "fast track" approach

to developing fusion energy source, shown for example in reports at: http://fire.pppl.gov/eu_fast_track_101201.pdf and http://www.naka.jaeri.go.jp/ITER/ITERsymposium/Bolt.pdf .

Because of the significant challenges associated with structural materials applications in these advanced nuclear energy systems, the Workshop on Advanced Computational Materials Science: Application to Fusion and Generation IV Fission Reactors was convened by the U.S. Department of Energy's Office of Science and the Office of Nuclear Energy, Science and Technology to ensure that research funded by these programs takes full advantage of ongoing advancements in computational science and the Department's investment in computational facilities. In particular, participants in the workshop were asked to:

(1) Examine the role of high-end computing in the prediction of materials behavior under the full spectrum of radiation, temperature, and mechanical loading conditions anticipated for advanced structural materials that are required for future Generation IV fission and fusion reactor environments, and

(2) Evaluate the potential for experimentally-validated computational modeling and simulation to bridge the gap between data that that is needed to support the design of these advanced nuclear technologies and both the available database and data that can be reasonably obtained in currently-available irradiation facilities.

An international panel of expert materials and computational scientists was invited to participate in the workshop. The workshop program is provided in Appendix A and the final makeup of the panel is listed in Appendix B. In addition to the panel, a number of interested scientists attended as observers and were given the opportunity to contribute to the workshop discussion. The list of observers is also included in Appendix B.

The workshop discussion focused on metals and alloys that will be used in the regions with the greatest potential for radiation-induced effects to occur, i.e. materials close to the fission reactor core or the fusion plasma. This limited scope does not imply that these are the only materials issues that need to be addressed for the successful implementation of Gen-IV or fusion reactors. Ceramics and ceramic composite materials and graphite will be used in the highest temperature, and some high neutron flux, regions of fission reactors such as the NGNP. Ceramics and composites will also see service in fusion reactors, both as structural components and as insulators in regions where radiation will lead to property degradation. In addition to the need for structural alloys, a number of other special purpose materials such as tungsten, copper, or $\mathrm{SiC}$ may see service in high heat flux, plasma-facing components. In some cases, the radiation damage issues for these materials are similar to those addressed in the workshop, but others also arise. These include radiation-induced conductivity and changes in dielectric properties for insulators, and sputtering for plasma-facing components. Thus, the workshop's focus on structural 
materials in relatively high neutron flux regions should be seen as an expedient, necessary evil due to the limited time available.

Structural materials proposed for use in conceptual designs for Gen-IV and fusion reactors include variants of complex ferritic-martensitic steels, superalloys, vanadium alloys, carbon-carbon composites and silicon carbide composites. Potential coolants include helium, water, sodium, lithium and lithium compounds, lead alloys, and molten salts. The primary method currently envisioned to provide high strength and fracture toughness as well as appropriate radiation resistance in hightemperature metallic components involves incorporating a high density of nanoscale second phase features to serve as point defect recombination centers and for helium trapping. However, these nanoscale features are intrinsically unstable under irradiation due to ballistic dissolution and other nonequilibrium processes, such as radiation induced solute segregation and radiation enhanced diffusion. Thus, a key challenge is to design materials for which the duration of the transient non-equilibrium stage is greatly increased. For this approach to be successful, the metastable nanoscale features must be maintained to damage levels approaching 200 displacements per atom (dpa). Understanding the stability of these features requires development of models that couple dissolution, segregation, and diffusion in complex systems. While the basis for components of such a complex model exist, the ability to integrate modeling components is still lacking.

The unique aspects of the DT fusion reactor environment, and the differences from fission reactors, predominantly arise from the differences between the DT and fission neutron energy spectra. Fission neutrons are born with a broad spectrum of energies peaking near $0.65 \mathrm{MeV}$ (the flux at $10 \mathrm{MeV}$ is reduced by a factor of 350), whereas the DT fusion neutron spectrum is essentially mono-energetic at 14.1 MeV. Although the energy of the fusion neutrons is quickly reduced by collisions with the materials that comprise the reactor structure, materials nearest the plasma are exposed to a neutron energy spectrum that is significantly different from that obtained in fission reactors. The effect of these higher energy neutrons is two-fold. First, fusion neutrons will obviously produce higher energy displacement cascades than the lower-energy fission neutrons. Based on coordinated modeling and experimental studies performed over the past 15 years, it appears the defects produced by fission and fusion cascades will be qualitatively similar and that displacement cascade damage differences can be accounted for using the well-established dpa unit of exposure.

The second effect of higher energy neutrons in the fusion spectrum is enhanced nuclear transmutation reactions [1], due to the existence of nuclear reactions with threshold energies between 2 and $15 \mathrm{MeV}$ in many structural materials. The production of solid transmutants (particularly surfaceactive impurities) can alter alloy properties. The transmutation products of greatest concern are hydrogen and helium because these gases can significantly impact microstructural evolution and be synergistic with displacement damage phenomena. Although a variety of theoretical and experimental techniques have been applied to examine the role of helium on microstructural stability and mechanical property changes, key aspects of the behavior and effects of helium remain unresolved. As a simple example, helium is known to stabilize small radiation-induced vacancy clusters. Depending on the ratio of helium to displacement production, this stabilization can lead to either more or less void swelling $[2,3]$, and a significant reduction in creep rupture times and ductility due to enhanced grain boundary cavitation.

\section{References}

1. K. Ehrlich, Phil. Trans. R.. Soc. London A 357 (1999) 595.

2. V. Levy, D. Gilbon and C. Rivera, in Effects of Radiation in Materials, $12^{\text {th }}$ International Symposium, ASTM STP 870, F. A. Garner and J. S. Perrin, Eds., ASTM International, West Conshohocken, PA, 1985, p. 317.

3. R. E. Stoller and G. R. Odette, in Effects of Radiation in Materials, $11^{\text {th }}$ International Symposium, ASTM STP 782, H. R. Brager and J. S. Perrin, Eds., ASTM International, West Conshohocken, PA, 1982, p. 275. 


\section{Session I: Overview of Relevant Irradiation Environments}

\subsection{Objectives of Session I}

This opening session was intended to provide a description of the operating environments for structural materials applications in anticipated advanced fission (Generation IV) and DT fusion reactors. The goal was to highlight the similarities and differences among the various exposure conditions for the purpose of informing the discussion in the following sessions. Potentially useful experimental facilities such as test reactors and ion irradiation facilities were also discussed, as was the possible relevance of spallation neutron sources which provide a similar challenge to materials performance [1].

\subsection{Introduction to Irradiation Environments}

Generation IV and DT fusion systems are expected to supply energy in a sustainable manner to modern economies for centuries. In this context, the word energy primarily implies electrical generation and the delivery of process heat at high temperatures for purposes such as hydrogen production. In order to obtain the desired dramatic advances in performance over current fission systems, advanced fission and fusion systems will operate in regimes and environments that are beyond current nuclear industry experience, as well as most previous experience with developmental fission systems [2, 3]. Materials must perform at higher temperatures and for longer times, and to larger irradiation doses than required in current light water reactor technology. In additional, some advanced nuclear system designs propose coolants that are more aggressive than high-temperature water. For successful deployment of advanced fission and fusion systems, significant advances in material technology are required.

In many important ways, the materials for fusion and fission systems face similar temperature, radiation, and corrosion environments. Table 1-1 below provides a general comparison of the range of operating conditions expected in advanced nuclear systems. Even among concepts with different coolants, many application requirements have important similarities (such as temperature, stress, or neutron spectrum), prompting selection of similar materials, or classes of materials.

Table 1-1. Advanced fission and fusion operating conditions

\begin{tabular}{lcc}
\hline & Fusion & Fission (Gen-IV) \\
\hline Coolant & $\mathrm{H}_{2} \mathrm{O}, \mathrm{He}, \mathrm{Li}, \mathrm{PbLi}, \mathrm{FLiBe}$ & $\mathrm{H}_{2} \mathrm{O}(\mathrm{SC}), \mathrm{He}, \mathrm{Pb}, \mathrm{PbBi}, \mathrm{Na}$ \\
Particle Energy & $<14 \mathrm{MeV}$ & $<1-2 \mathrm{MeV}$ \\
Temperatures & $300-1000^{\circ} \mathrm{C}$ & $300-1000^{\circ} \mathrm{C}$ \\
Max displacement damage & $\sim 200$ & $15-200$ \\
He/dpa & 10 appm/dpa & $\sim 0.1$ appm $/ \mathrm{dpa}$ \\
Stresses & Moderate, nearly constant & Moderate, nearly constant \\
\hline
\end{tabular}

The materials of construction must demonstrate adequate mechanical strength, ductility, and toughness, must demonstrate adequate dimensional stability, must maintain adequate thermo-physical properties, and must have acceptable resistance to corrosion and stress corrosion attack from cooling media [4]. Experimental programs cannot cover the breadth of materials and irradiation conditions for the proposed fusion and Gen-IV fission reactor designs. A set of tools is required to assist in a material 
selection process that will be performed based on an incomplete experimental database and that requires considerable judgment to carry out the necessary interpolation and extrapolation. Modeling and microstructural analysis can provide the intellectual foundation for these important decisions.

Materials degradation from radiation starts with collisions between high-energy neutrons and lattice atoms and then develops into radiation-induced microstructures that degrade mechanical properties, dimensional stability, thermo-physical properties, and resistance to environmental attack. This is true in both fission and fusion systems. A set of computational tools that adequately describes radiation events and subsequent microstructural development and provides from this microstructure an accurate description of property changes in engineering materials would be a tremendously valuable tool for system designers. While developing these computational tools into a truly predictive capability is a difficult and time-consuming challenge, the knowledge gained from development of the individual model components will provide valuable insight to any on-going materials design effort. These models cannot be developed without a parallel set of validation experiments. The interplay between new experimental data and advanced theory or computational tools is critical to the development of accurate radiation response models.

While there are many similarities between fission and fusion systems, some important differences do exist. For example, the higher energy neutrons in fusion systems will lead to greater concentrations of the transmutation gasses helium and hydrogen. Additionally, certain Gen-IV concepts use different coolants and may respond to changes in microstructure in a unique manner. These differences do not dramatically change the types of computational tools that need to be developed. The tools need to encompass broad time and length scales from descriptions of primary damage formation all the way to bulk property changes. Properly done, this basic set of tools will be valid for both fission and fusion. The differences in environment will dictate only that a broader set of confirmatory experiments is required to ensure the models are valid over the entire operating regime of fission and fusion systems.

\subsection{Related Research and Development Issues}

In addition to a survey of the relevant irradiation environments, the members of this group also discussed the issue of materials needs for advanced nuclear energy systems from three different perspectives. Here their overview encompassed:

- Irradiation environments and the physical and chemical processes engendered by radiation damage

- Computational capabilities required

- Research and development and engineering objectives

The first perspective concentrated on the physical and chemical processes involved in radiation damage and in changes to relevant materials properties, and the second reviewed computational methods and how they map onto the physical and chemical processes that must be modeled and evaluated computationally. Finally, the desired outcomes were enumerated and an assessment was made of the nature and extent of information required to enable design of new reactor concepts without construction of many prototypes or extensive testing.

\subsubsection{Irradiation Environments and the Physical Processes Engendered by Radiation Damage}

The progress achieved during the last two decades in our fundamental understanding of radiation damage in solids has shown that there exists a unified theoretical description for the majority of the processes involved, and that these processes are essentially the same for most irradiation environments. It then follows that a complete theoretical formulation of all the processes and all the cause-effect relationships must be applicable to irradiation with neutrons, electrons, $\gamma$-rays, ions, and fission products. Conversely, results obtained from a particular irradiation environment must be useful 


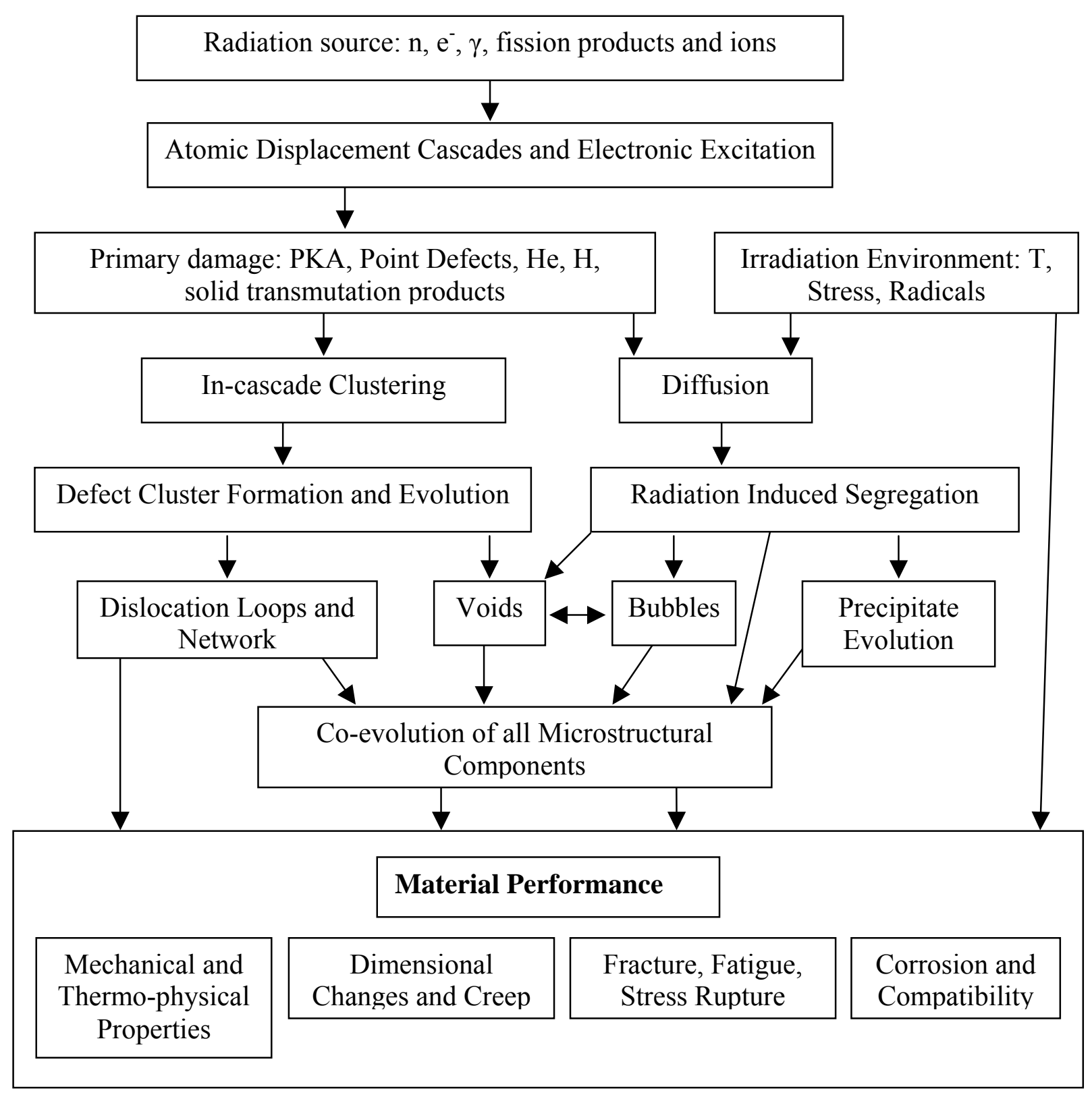

Figure 1-1. Schematic diagram of radiation damage processes

for all others. In the past, the complexity of radiation damage processes required simplified treatments tailored to a specific radiation environment, and the models so obtained were limited. Today, and even more so in the near future, advanced computational materials science can take full advantage of the universal theoretical description of radiation damage. Naturally, many of the processes need to be dealt with, be refined and validated, but the entire edifice can now be clearly recognized in its overall shape and scope. Figure 1-1 presents a rather compact rendition of this edifice. It shows that the radiation sources all produce primary recoil atoms via elastic collisions [5] that in turn produce collision cascades $[6,7]$, the sources for defects and defect clusters [8].

At the elevated temperatures existing in nuclear reactors, these defects diffuse, dissolve, and reaggregate in other forms throughout the material. These secondary defects evolve into a complex new microstructure, and in the process, change microscopic and macroscopic properties of the material. This 
picture holds for both metallic alloys and insulators. In addition, inelastic collisions are known to affect the chemical bonds of insulators, creating charged defects which will also diffuse and induce changes in related macroscopic properties.

\subsubsection{Computational Capabilities Required}

Many of the theoretical tools required have already or are being developed by a much larger group of experts active in many branches of solid-state physics and materials science. This will greatly benefit the smaller group of scientists active in the area of radiation damage. The tools can be grouped in four main classes that include different types of models as shown in the flow chart in Figure 1-2, along with their main output physical quantities.

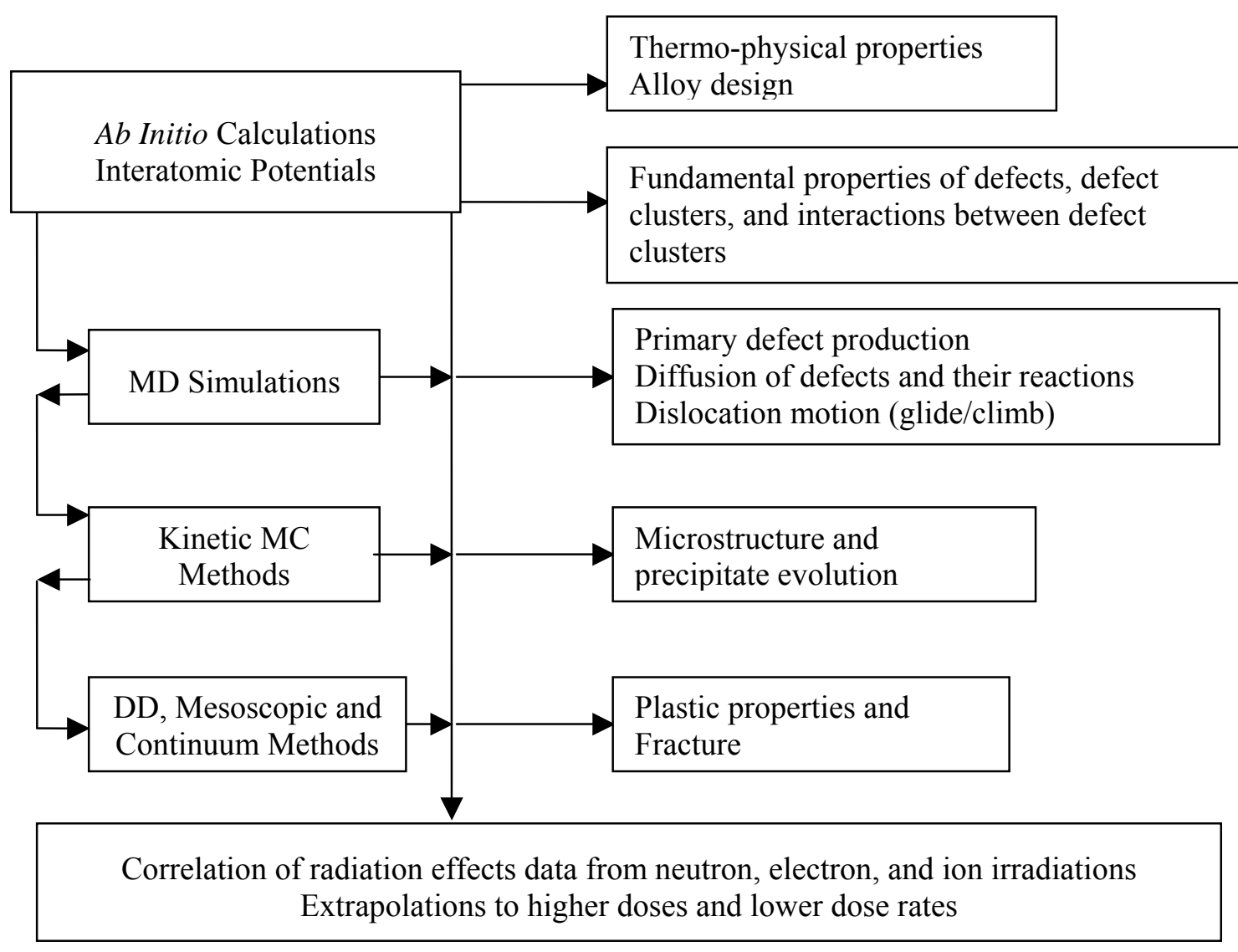

Figure 1-2. Summary of computational tools and their uses in radiation damage modeling

These models have typically been developed and applied to pure elements or simple dilute alloys. In contrast, the high in-service temperature and radiation levels typical of nuclear application require multi-element concentrated alloys with optimized chemical compositions and microstructures. The reliability of the prediction of multiscale modeling for nuclear materials will thus depend very much on the capability of the nuclear materials community to select, develop, and apply models that accurately treat the critical components of microstructural evolution in complex alloys and relate that microstructural evolution to specific changes in macroscopic properties. An in depth analysis of the present capabilities of these modeling tools and their future development are given in Sessions II-V.

\subsubsection{Research and Development and Engineering Objectives}

The computational tools must provide the basis for: (a) re-assessing existing data, (b) optimizing new experiments in test reactors or in a dedicated neutron source $(14 \mathrm{MeV})$, and (c) 
interpreting the obtained data and reliably extrapolating them to anticipated in-service conditions, the domain of which is always broader than those achieved in experiments. The ultimate goal is to use the experimentally validated models to enable scientists to improve existing materials or to design better materials.

For engineering purposes, the computed and measured property changes need to be codified into databases or constitutive relationships that an engineer can use to design a nuclear energy system, assess its performance, and determine its ultimate lifetime.

\section{$1.4 \quad$ Near-term Research Priorities}

Although there is a vast number of physical changes induced by irradiation, some phenomena are either already understood at a sufficient (albeit rudimentary) level for evaluating feasibility issues, or are not critical determining factors in establishing the feasibility of using materials in irradiation environments. Conversely, there are several fundamental radiation effects phenomena that critically need improved understanding in order to assess candidate material options. These include defect production and evolution in alloys and ceramics (as opposed to pure metals), coupled solute-defect diffusion processes in concentrated alloys, microstructural evolution of coupled point defect sinks (e.g., voids and dislocations), and the effects of point defect clusters, surface active transmutant impurities and helium on localized deformation and fracture toughness. These are briefly discussed in the following section.

A firm understanding of the fundamentals of defect production and evolution in alloys is essential for evaluating the microstructural stability of candidate engineering materials in experimentally unexplored irradiation conditions. The current defect production modeling efforts have been largely limited to pure metals such as $\mathrm{Fe}$ and $\mathrm{Cu}$. It is well known from fundamental experiments performed with ion and fission neutron irradiation sources that the production and evolution (annihilation, clustering) of point defects and defect clusters is significantly different in alloys compared to pure metals. This appears to be due to enhancement or suppression of point defect recombination within the displacement cascade as well as solute effects on point defect mobility and trapping at solute precipitates. Subtle differences in minor alloying elements in complex engineering alloys have been observed to have a pronounced effect on their stability during neutron irradiation. As a first step toward accurate modeling of prospective engineering alloys, suitable interatomic potentials need to be derived for important binary alloy and ternary systems including $\mathrm{Fe}-\mathrm{Cr}, \mathrm{Fe}-\mathrm{Ni}, \mathrm{Fe}-\mathrm{He}$, and $\mathrm{Fe}-\mathrm{Cr}-\mathrm{Ni}$. To properly account for the behavior of steels, the influence of $\mathrm{C}$ and $\mathrm{N}$ also need to be included. An improved understanding of helium effects in irradiated materials is of particular importance for fusion energy, since recent simulation experiments suggest that fusion-relevant helium generation during neutron irradiation may have severe deleterious effects on the dimensional stability and fracture toughness of current candidate alloys. Similar improvements in the fundamental understanding of defect production and evolution in irradiated nonmetals such as ceramic insulators are also needed. In particular, the conditions for production of defects in nonmetals by pure ionizing radiation (radiolysis and high electronic stopping power processes) need to be accurately understood so these effects can be separated from knock-on collision effects. The migration and annihilation processes of charged and uncharged point defects in nonmetals also need to be understood.

There is a strong need to develop improved physically based models and simulations of radiation-induced and -enhanced segregation processes. The current segregation models used by many researchers are based on models originally developed in the 1970s (solute drag and inverse Kirkendall effects). Dramatic improvement was achieved in the 1990s by the introduction of models that used atomistic information to account for diffusion in concentrated alloys [9]. However, many of the key physical parameters in these models are not well known, including the binding energies and migration energies of various solute-point defect complexes as a function of the local composition. On a broader level, the kinetics and mechanisms of diffusion in concentrated alloys in the presence of solute gradients are not sufficiently known. This opens the possibility that new solute-defect coupled diffusion processes 
may occur under irradiation that are not covered by the rudimentary solute drag and inverse Kirkendall models. As a first step toward development of improved point defect-solute models, the vacancy formation energy and migration energy should be determined as a function of solute content (including carbon) in iron and other important material systems. The role of dumbbell interstitials, particularly mixed solute-solvent dumbbells, also requires further investigation.

Current models of radiation-induced void swelling treat voids and network dislocations as separately evolving systems that are linked only by the effect of void and dislocation sink strengths on the arriving point defect fluxes. However, experimental studies have found that dislocations thread voids in many cases. Therefore, it may not be appropriate to model void and dislocation evolution during irradiation as weakly interacting systems. Kinetic Monte Carlo studies of microstructural evolution in the presence of coupled point defect sinks (voids/dislocations) would be valuable to provide guidance on the importance of this coupling. The effect of helium generation on this coupling could be subsequently investigated. The effect of chemical segregation on the development of microstructural features should also be studied.

The advent of 3-dimensional dislocation dynamics during the past decade has been a major achievement for modeling numerous important materials phenomena. However, dislocation climb processes have not yet been modeled in 3-dimensional dislocation dynamics codes. Inclusion of the various modes of dislocation climb would enable 3-dimensional dislocation dynamics to be quantitatively applied to investigations of microstructural evolution of irradiated materials. A further challenge is how to develop the appropriate linkages of 3-dimensional dislocation dynamics to atomic scale and mesoscale regimes so that multiscale phenomena such as fracture toughness can be appropriately modeled.

There are numerous deformation and fracture phenomena that need improved understanding. For example, there is current debate within the research community whether the long-standing connection between cleared dislocation channels and low tensile elongations is a bona fide cause and effect in metals irradiated at low temperatures. Improved multiscale models are needed to analyze potential causes of the low ductility in irradiated metals and to identify possible metallurgical solutions to mitigate this effect. The effects of point defect clusters and helium bubbles on crack formation and propagation also need to be investigated. It is uncertain whether there are any new He-enhanced fracture toughness degradation mechanisms at low temperatures beyond normal matrix hardening effects. Development of physically realistic multiscale models, combined with experimental studies, could provide important insight in this critically important area. Similarly, the potential effect on deformation and fracture of surface-active impurities produced by neutron transmutation reactions should be investigated. There is a largely incomplete understanding of the physical phenomena responsible for the poor elevated temperature ductility of superalloys during irradiation. If the physical processes responsible for the low ductility of superalloys irradiated at high temperatures could be identified and mitigated, superalloys would be attractive candidates for fission Gen-IV and fusion systems. Finally, despite nearly 40 years of research, a comprehensive model for high temperature helium embrittlement is not yet in place. High temperature helium embrittlement is anticipated to limit the maximum allowable doses and temperatures for structural alloys in fusion and Gen-IV fission reactors, and appropriate multiscale models and experiments should provide guidance for metallurgical improvements to increase the dose and temperature limits.

\subsection{Validation and Verification of Models}

Validation and verification of the predictions made by computational models can be performed using either established benchmarked computational codes or experimental data. Computational benchmarking and validation/verification of model predictions can also be obtained by comparing the results obtained from several different computer codes or techniques applied to analyze a similar scenario. Direct validation and verification of model predictions can be obtained in many cases by 
performing appropriate experiments. The experimental data for model validation and verification can be grouped into five categories:

(1) Mining of old data: Previously published experimental data represents an important resource that should be fully utilized in order to minimize costs associated with mounting new experiments. Experimental studies on fundamental point defect or materials properties are of particularly high value. Appropriate caution must be applied in extracting experimental data from published studies, since most data have been processed using models of varying degrees of robustness. In some cases, previously reported experimental data may need correction to account for deficiencies in the models originally used to analyze the test results.

(2) Mining of old reactor materials: Archive irradiated specimens from prior nuclear energy programs should be preserved wherever feasible for future experimental study. Due to rising costs for storage and disposition of radioactive materials, there is increasing pressure to dispose of specimens that are not of immediate value. These specimen storage costs must be weighed against the costs and technical value of performing new specifically designed experiments to obtain the same data. Of course, in many cases new experiments may provide opportunities to obtain data of significantly improved quality.

(3) Irradiation experiments using ion implantation, electrons, fission neutrons and spallation neutrons should be designed and performed to elucidate or quantify specific mechanisms that are building blocks in the multiscale modeling framework. These mechanistic experiments span the range from single-variable tests to multiple-variable synergistic phenomena studies. In many cases, specifically designed experimental techniques (such as tritium doping or He injector foils for studying He effects) may provide important insight on the controlling physical mechanisms.

(4) Prototypic irradiation experiments enable the opportunity to systematically investigate single-variable or synergistic multiple-variable effects on material coupons under environmental conditions similar to the intended application (e.g., DT fusion neutron spectrum or supercritical water irradiation assisted stress corrosion cracking phenomena). These prototypic tests are traditionally of very high value for confirming the predictions of computational models and establishing the safety basis for gaining licensing approval of reactors.

(5) Component testing: Integral irradiation experiments on reactor components (such as fusion reactor blanket modules) should eventually be tested in a dedicated facility. Integral tests of this kind are essential to validate that all the detailed models of materials performance are integrated in a seamless manner, and more importantly, that synergistic effects and phenomena are captured and well understood before a new reactor is built. Component testing is generally not initiated until the separate-effects data obtained from coupon testing in prototypic environments are fully analyzed using appropriate models.

In addition to providing predictive insight on potential physical mechanisms, computational models can provide important hindsight by explaining archive experimental results. Appropriate computational models are essential for obtaining maximum usefulness of data obtained in nonprototypic irradiation environments.

Several categories of experimental data are needed for computational model validation and for licensing. The most fundamental information is associated with point defect and defect cluster properties (formation, migration and binding energies, relaxation volume, 1-D vs. 3-D diffusion characteristics, etc.). These fundamental properties generally cannot be obtained directly from experiment, but instead are inferred from experimental observations by utilizing appropriate models. 
There are numerous classic examples in the literature where published fundamental data have required subsequent correction due to neglect of important physical phenomena in the original data analysis. Important experimental tools for acquiring fundamental radiation effects data on point defects and defect clusters include a variety of X-ray diffraction techniques, electrical resistivity methods, stored energy, positron annihilation spectroscopy, density, high resolution and microanalytical transmission electron microscopy, Mössbauer spectroscopy, optical spectroscopy, elastic moduli tests, and a variety of mechanical property measurements. The microstructural evolution of irradiated materials involves integration of multiple phenomena over extended time periods and length scales. Experimental observations obtained from electron microscopy, X-ray diffraction, positron annihilation, and other microstructural tools are valuable for validating and calibrating multiscale computational models. The microstructural evolution drives a number of important changes in physical and mechanical properties that can be used in concert with microstructural investigations to experimentally verify and validate multiscale model predictions. Important mechanical properties for engineering purposes include tensile elongation, tensile strength, thermal and irradiation creep, fracture toughness, fatigue strength, and fatigue crack growth rate.

\section{References}

4. L. K. Mansur, A. F. Rowcliffe, R. R. Nanstad, S. J. Zinkle, W.. R. Corwin, and R. E. Stoller, "Materials Needs for Fusion, Generation IV Fission Reactors, and Spallation Neutron Sources Similarities and Differences, 11 th International Conference on Fusion Reactor Materials, Kyoto, Japan, December 2003, to be published in J. Nucl. Mater.

5. A Technology Roadmap for Generation IV Nuclear Energy Systems, Report No. GIF002-00, December 1, 2002 (http://nuclear.gov).

6. E. E. Bloom, S. J. Zinkle, and F. W. Wiffen, "Materials to Deliver the Promise of Fusion Power," 11th International Conference on Fusion Reactor Materials, Kyoto, Japan, December 2003, to be published in J. Nucl. Mater.

7. Higher Temperature Reactor Materials Workshop, ANL-02/12, Argonne National Laboratory, August, 2002.

8. L. R. Greenwood, J. Nucl. Mater. 216 (1994) 29.

9. R. S. Averback, J. Nucl. Mater. 216, (1994) 49.

10. R. E. Stoller and L. R. Greenwood, J. Nucl. Mater. $271 \& 272$ (1999), 57.

11. L. K. Mansur, J. Nucl. Mater. 216 (1994) 97.

12. M. Nastar, Defect and Diffusion Forum 194-199 (2001) 171. 


\section{Session II: Current State of the Art in Relevant Multiscale Computational Materials Science Models}

\subsection{Objectives of Session II}

The subpanel responsible for Session II was charged with characterizing the adequacy of the current state-of-the-art in multiscale computational materials science models for the purpose of simulating radiation effects in structural materials. The participants determined that it will be necessary to set in place over the next five-to-ten year period a suite of computational tools to model, predict and guide assessment of ferritic steels under relevant conditions of damage, helium generation, temperature, time and stress. The crucial properties for simulations of reactor materials radiation damage are (a) defect creation; (b) defect migration and interaction; (c) boundary structure and segregation effects; (d) dislocation behavior under stress; and (e) phase stability. It is essential that the modeling techniques be robust and versatile enough to treat other materials and other problems that enter the frame over the time period. Furthermore, the tools must be able to guide future experiments as well as be informed by them.

This section devotes considerable attention to atomic-scale methods because radiation damage in reactor components is created at this scale and the evolution of damage is controlled by processes on this scale. Furthermore, recent advances in such computationally-based methods offer exciting opportunities for prediction of materials properties in situations where experimental information is unavailable. Nevertheless, the scope for computational materials science to tackle important problems at the continuum scale must not be lost sight of: yielding, creep and fracture are examples of processes where simulation is required across all scales. In terms of increasing length and time scales, the relevant modeling aspects considered include $a b$ initio methods, interatomic potentials, molecular dynamics and other methods of treating materials discretely at the nano-to-micro scale, continuum-based modeling and developments for simultaneous linking of length and time scales. Current developments are discussed in the following sections, but first we consider some of the current issues involved.

\subsection{Overview of Current Issues}

Molecular dynamics (MD) is the most widely used method for atomic-scale simulation. Much of our current understanding of, for example, primary damage in cascades, point defects and their clusters, dislocation core properties and crack-tip processes, comes from MD simulation. In MD, the classical equations of motion are integrated to obtain dynamical evolution of a system of atoms. Accurate integration requires time steps in the femtosecond range, limiting the total simulation time to less than a microsecond on today's processors. Direct MD is a powerful probe, giving the classically exact dynamical evolution of a system for a given interatomic potential. It provides for determination and prediction of material properties at the atomic scale and, since higher-level models for materials evolution (kinetic Monte Carlo (KMC), dislocation dynamics, phase field models, rate theory, etc.) involve assumptions and approximations about properties, it offers a benchmark for their verification. Advances in interatomic potentials described below in section 2.3 will mean that MD simulations can be compared directly to experiment. Two possible exceptions to this are light atom (hydrogen or helium) diffusion below room temperature, where quantum corrections to the dynamics start to become important, and dynamics during the thermal spike stage of a cascade, when non-Born-Oppenheimer effects omitted from MD become important (e.g., thermal transport via electron-phonon coupling in a metallic system or electronic excitations in a covalently bonded system).

Computer memory sets the upper limit for the number of particles, i.e. the system length scale. Fortunately, the static or dynamic properties of relatively small subsystems often determine the macroscopic materials behavior. This explains the widespread use and success in materials science of electronic band structure calculations, molecular statics and MD simulations. However, MD parallelizes well via spatial decomposition, offering an easy path to extend the length scale. Simulations of a million atoms are routine, billion-atom simulations have been performed, and it should be possible to simulate 
$\sim 10^{11}$ atoms (= one-micron cube of $\mathrm{Fe}$ ) for approximately a microsecond on the next-generation leadership-class machine. Increasing model size will allow unprecedented direct examination of phenomena, such as multi-dislocation interactions and fracture. This means, for instance, that displacement cascades created by primary recoils with energy up to a few $100 \mathrm{keV}$ can be simulated, although electronic effects (excitation and electron-phonon coupling) are likely to remain difficult to incorporate in MD models. Since subcascade formation is common in most metals at the higher energies in this range, the use of Binary Collision Approximation (BCA) codes, tuned to MD results at overlapping energy, is expected to be fruitful.

Time scales accessible to MD are more problematic. The calculation of the energy and interatomic force is by far the most expensive part of an MD simulation, so complexity in the interatomic potentials should be avoided. Model size is currently limited to about a thousand atoms if accurate quantum mechanically derived forces are used and larger systems mentioned in the preceding paragraph have to be described by more approximate force fields. Total simulation time and/or model size is expected to continue to increase proportionally to single-processor speed, but physical limitations in processor design will cause this to flatten out in the next 5-10 years. Consequently, direct MD simulations of million-atom systems may never reach one millisecond, even with simple potentials. As many important activated phenomena (diffusion, defect clustering, void growth, grain growth, dislocation motion, creep, etc.) are active on time scales orders of magnitude beyond this, this poses a critical problem. Thus, methods of speeding up MD simulation are required. We address this in section 2.4. These techniques are not applicable to many classes of problem, however, and then it is necessary to use either the MC or continuum methods described below.

The nature of dislocation motion within the microstructure of an irradiated material is a major factor to be considered for assessing the in-service integrity of reactor components. Motion can occur as a result of either applied stress, internal stress or chemical stress due to point defects, and gives rise to phenomena as varied as uniform flow, localized yielding, hardening, crack tip plasticity and creep. Until recently, most models of dislocation glide and climb in the presence of defects created by damage fluxes were based on the elasticity theory of dislocations, with line shape under stress estimated using the line tension approximation. This approximation neglects the effects of self-interaction between different parts of the line, which, for dislocations overcoming obstacles, can have an important influence on the flow stress. A dislocation with self-stress can be modeled as a flexible line constructed of piecewise segments. Although self-stress was first treated in this way over 30 years ago [1], it was only possible to consider a few hundred segments for a few tens of iterations. Ideally, 3D dislocation dynamics (DD) models of plasticity on the micro-meso scale require the simulation of $0.1-100 \mathrm{~mm}$ of line length (for a dislocation density of $10^{14} \mathrm{~m}^{-2}$ ), and thus contain typically $10^{5}-10^{8}$ segments. They should also be able to achieve a total plastic strain at realistic strain rates (e.g. $10 \%$ at $10^{-6} \mathrm{~s}^{-1}$ ). These aims cannot be met at the moment. Hence, new, more efficient techniques for computing the field of segments and their interactions with each other and damage microstructure are required. As discussed in section 2.5, these, coupled with expected advances in computing power, will enable the goals to be met.

Continuum-based DD models have uncertainties associated with singularities in the dislocation stress field and the loss of validity of the elasticity description at the atomic scale. These limitations do not apply to MD simulation of dislocation behavior. Hence, MD modeling can provide detailed information on obstacle forces [2] and dislocation core effects [3] for incorporation in larger scale DD simulation. However, the requirements on MD for treatment of plasticity at the length and time scales of interest are many orders of magnitude beyond current computing power. For example, limits on simulated time restrict the modeling of dislocation glide to strain rates of about $10^{6}$ per second, whereas rates a billion times smaller are of primary concern. For dislocation-controlled creep, the rates of interest are orders of magnitude smaller again. Thus, the limitations and benefits of using this method have to be recognized. Current work is discussed in section 2.5.

It is necessary to ensure that the atomic system is not dominated by artificial boundary effects, i.e. it should behave as a representative part of the surrounding macrosystem. For instance, band 
structure theory utilizes periodic boundary conditions to mimic an infinite solid. In more general situations, defects break translational symmetry and more complicated boundary conditions are needed. This problem is closely related to the length scale limitations. Except for the case of free clusters, boundary effects such as stress or strain, temperature or heat flux have to be applied to the atomistic system. In addition, energetic events, e.g. interaction of high-energy atomic particles with solids, create pressure waves, which, without careful choice of boundary conditions, can cause inaccuracies in the evolution of the subsystem.

Simultaneous coupling of different methods with appropriate spatial resolution and accuracy can overcome such problems and also raise the limits of atomistic system size. The multiple length scales are covered by continuum mechanics (for the macroscopic part of the material), computationally inexpensive interatomic potentials (bridging the mesoscale where the atomic motion has to be resolved) and quantum-derived forces (for high accuracy in the nanoscale parts where strong non-equilibrium processes prevail). Radiation damage and crack formation are examples where hybrid schemes can be valuable, i.e. accurate forces are required close to a strong local disturbance, its neighborhood can exhibit thermal or mechanical disorder for which simple empirical forces are adequate, and the longrange response can be supplied by a surrounding continuum. The general issue of coupling across the scales is considered further in section 2.5.

The evolution of microstructure and properties of materials under intense energy input may be controlled by essentially kinetic phenomena and so not understood in thermodynamic terms only. The main methods used currently to predict the kinetics are, in order of increasing space and time scales, the various forms of KMC (mostly on Rigid Lattice, Event-based or Object-based), Self-consistent Mean Field, Phase Field, and Cluster Dynamics (Rate Theory) models. In order to develop robust, physicallybased models for predicting the kinetic pathways and "dynamic phase diagrams," it is necessary to calculate accurately the populations and elementary properties of point defects, and determine the detailed mechanisms of atomic migration on which the evolution models are based. Recent developments of first principles methods, coupled with fast computation techniques to explore configurations in systems of increasing size, allows complex defects and alloys to be considered [4,5]. The coupling of first principles calculation with kinetic models is now being validated with success against experimental data, thereby demonstrating the robustness of this approach. It is described further in section 2.7 .

\subsection{Interatomic Potentials}

\subsubsection{Current status}

MD and MC simulations require an interatomic potential that represents the energy and forces associated with a configuration of atoms in a real material. To be usable for complex geometries and/or statistical averages, the potential needs to be computed rapidly. Many interatomic potentials have been developed and are reported in the literature, but none are necessarily adequate for simulation of the wide range of properties required for reactor materials. With pair-wise interactions some are necessarily wrong. With many-body potentials (used here as a generic term covering glue, Finnis-Sinclair, embedded atom, modified embedded atom and effective medium theory potentials) many can be fitted provided "correct" values are available. These types of potentials have been the "state of the art" for twenty years.

Historically, there has been an insufficient database for robust potential fitting. Not all this data is available experimentally for parameterization and verification of potentials. Recent renewed interest in interatomic potentials is based on the ability of $a b$ initio calculations to provide this missing data with teraflop machines verification of predictions is finally possible. Where tested against new data, existing potentials have generally proved disappointing. Problems include incorrect self-interstitial configuration and energy, and no satisfactory description of the austenitic-ferritic transition. Some problems can be traced to parameterization of the potentials and addressed by simple 
reparameterization. Others, such as the absence of a physically sensible treatment of magnetization, point to more fundamental problems in the many-body potential concept.

Most effort for metallic phases has focused on elemental materials. Potentials for some multicomponent systems have been developed, but their predictive capability is typically disappointing. Reasonable models for the mission-critical helium impurities exist, the inertness of helium making its behavior in MD somewhat insensitive to parameterization. There are two challenges in the development of potentials for alloy systems. First, there is generally much less data available, though this can be rectified through the use of $a b$ initio methods. Second, the appropriate functional forms are not as well developed. Most potentials are based on simple pictures of bonding. In alloy systems, the nature of the bonding is inherently more complex, suggesting that more sophisticated potentials are needed. Nonmetallic impurities, such as carbon and phosphorus, are more problematic.

The advent of $a b$ initio calculations has allowed more accurate verification procedures to be applied to existing models, and highlighted some serious flaws. The prospects for rectifying these are good: some are simply a matter of reparameterization and extension to systems where insufficient underpinning data existed previously [6,7]; others require implementation of new models already proposed [8]; some may require the development of new approaches. The primary resource requirement for these developments is manpower, inspiration and stronger interactions between research groups to understand one another's needs.

\subsubsection{Where do we need to go with interatomic potentials?}

Discussions at the workshop highlighted three areas regarding interatomic potentials that warrant attention for further development.

\subsubsection{Validation and reparameterization of existing many-body models.}

The technology for validation based on $a b$ initio simulation is mature. A database of structures and migration paths for point defects with and without impurities needs to be created. (This will also benefit calculation at the KMC level). Efficient fitting techniques based on genetic algorithms can be employed to assist parameterization. Crucial data not available currently $a b$ initio includes defect migration mechanisms, dislocations core states and defect-dislocation interactions, and will require petaflop computing.

\subsubsection{Development of better models for magnetism and alloying.}

It is possible to create potentials with more complex functional forms than currently, but with similar computational complexity. Two new types have been proposed in Europe and should be exploited by the program.

The two-band model represents the electronic structure on each atom by a single, local, variational parameter $\mu$. This has been applied to describe the volume-collapse transition in Cs where $\mu$ represents s-d transfer [8]. The model has potential applicability to ferritic steels where $\mu$ is the magnetization, and to materials where f-localization is important. The functional complexity does not result in additional computational complexity thanks to the Hellmann-Feynman theorem, which means that the force can be evaluated without differentiating $\mu$.

Composition-dependent potentials offer the possibility to incorporate non-local Fermi-surface effects by making the potential dependent on the global as well as local electron density. This addresses such anomalies as the multiple bcc-fcc-hcp phase transitions observed with many short-range potentials under pressure. Here the functional form throughout the simulation is modified according to the composition and density of the MD simulation cell. This is practical since almost all MD simulations conserve particle number. There are some problems with constant pressure MD, since a fictitious term appears in the pressure - however since all applications of constant pressure MD already have a fictitious mass in the Lagrangian this issue should be tractable with perturbative corrections akin to the 
Pulay stresses encountered in plane-wave DFT. Simple composition-dependent potentials have been applied to dilute $\mathrm{Fe}-\mathrm{Cr}$ alloys which exhibit an oscillatory alloying energy that cannot be reproduced with conventional many-body potentials.

These two models, and a reawakened interest in new model complexity, offer tremendous promise for extending existing MD models to the complex multi-component steels and to properly understanding the atomic basis of the role of minority additives in determining bulk properties. This in turn opens the possibility of guiding development of materials, reducing the number of samples required to be synthesized for experimental testing.

\subsubsection{Interaction with applied mathematics community}

The underlying challenge is to develop a computationally convenient representation of the energy and forces between atoms that can be computed from $a b$ initio methods. This can be viewed as an applied mathematics problem of finding a reliable representation of a known complex function. A clear opportunity exists for collaborations between the physics and mathematics communities to develop novel approaches to the challenge of representing the Born-Oppenheimer surface for a variety of applications.

\subsection{Molecular Dynamics: Accelerated Molecular Dynamics Methods}

It seems clear that many of the important phenomena in irradiated materials will have to be studied over the next few years by classical MD with computationally-simple interatomic potentials, i.e. millions of atoms for times on the order of a nanosecond, or thousands of atoms for perhaps a microsecond. However, in the last few years, a new class of methods [9] aimed at the micro-to-millisecond time scale has emerged. Their goal is to propagate the system rapidly from state to state in a correct fashion without introducing assumptions or prior knowledge about the possible mechanisms for diffusion or reaction. This is a departure from the KMC approach, for in accelerated MD methods the system finds its own way out of each state without prior specification of the mechanisms. Applying statistical mechanical concepts, this escape process is stimulated to occur more quickly, but with little, or no, corruption of the probabilities for escape along any given path. In this way, a volume of material can be evolved forward from state to state out to much longer times than are possible with standard MD. In the temperature accelerated dynamics method (TAD) [10], with a few controlled approximations the computational speedup ('boost factor') can reach into millions [9] when the barriers are high relative to the temperature, i.e. TAD can reach time scales of seconds and beyond on a single processor. This has been demonstrated for vapor-deposited film growth of copper [9] and radiation annealing of low-energy cascades in $\mathrm{MgO}$ [11]. For systems with persistent low barriers, which are common for realistic cases, however, the boost factor is more modest and improving on this limitation (without introducing significant approximations) is an important area of ongoing research. In the parallel-replica dynamics (PRD) method [12] time is parallelized. PRD is very general, requiring only that the reactive processes be first-order (exhibit exponential first-passage statistics), and that transitions can be detected. An appealing feature of PRD is that it gives exact state-to-state dynamics, with parallel speedup, if properly implemented. There is no assumption of Arrhenius behavior, nor even that there is an energy barrier associated with reactive events, i.e. the bottleneck can be purely entropic. Inter-processor communication requirements are minimal; parallel efficiency can be maintained up to the point where the time between reactive events has been collapsed to the order of the correlation time (a few ps for a transition metal system). Thus, for events separated by a few microseconds, a computational boost factor on the order of a million could be achieved on a million processors. However, as noted above, the more typical case is that low barriers in the system limit this boost.

Besides this boost limitation, the other key remaining issue for these methods is the accessible length scale. The TAD method in its present form becomes inefficient for systems larger than $\sim 1000$ atoms due to the fact that the computational work scales super-linearly with system size. For the PRD method, the system size matters only in that for larger systems there tend to be more available activated 
processes, with decreasing average time between events and maximum possible parallel boost. The lowbarrier and length scale issues are the subject of ongoing research, and should be considered for future research funding.

These new methods offer opportunities for unprecedented investigations on the next generation of massively parallel computers. One example would be parallelizing both for electronic structure, and then, multiplicatively, for PRD. If the electronic structure-based MD could reach, say, $200 \mathrm{ps,} \mathrm{then}$ parallel-replica dynamics could follow the system through about 40 transitions. This could allow the first view of post-cascade dynamics on the microsecond time scale with full electronic structure accuracy.

\subsection{Dislocation Dynamics}

\subsubsection{D dislocation dynamics}

Dislocation Dynamics (DD) simulations approach is becoming an increasingly important tool in the computational materials research $[13,14]$. Over the last several years there have been considerable advances both in the general DD methodology and in its implementation on massively parallel computers. The uniqueness of DD is its ability, on one hand, to incorporate the atomistic mechanisms of dislocation motion and interactions (among dislocations and with other elements of material microstructure) and, on the other hand, to directly compute the plastic strength of crystalline materials. Thus, DD provides a much-needed bridge between the length and time scales of the fundamental physical mechanisms and the length and time scales relevant for engineering applications.

Three major challenges for further development of the DD approach are that (1) the unit mechanisms of dislocation motion and interaction are multiple and complex, (2) one needs to trace the evolution of very large groups of dislocations over extended periods of time while accessing the length and time scales relevant for microstructure evolution and its effects on yield, flow, strain hardening and strain localization, and (3) development of connections between DD and continuum plasticity theory. Recent achievements of $4-10 \%$ of plastic strain in a direct DD simulation suggest that the approach is on its way to becoming computationally efficient, through the use of massively parallel computing and more advanced numerical algorithms. The most serious of the remaining computability challenges is to scale DD simulations down to lower straining rates $\left(\sim 10^{-5}\right)$ and yet maintain their computational efficiency. The problem here is to be able to handle multiple time scales observed in the evolution of large dislocation populations, where periods of relative calm (slow motion of lines) are interspersed with bursts of very high activity over which small groups of lines moves very fast and experience multiple collisions.

Parallel DD implementations face their own problems. The first one is a programming challenge: the DD models deal with the ever-changing topology of dislocation lines and line networks requiring rather complex data structures and careful bookkeeping. On parallel machines, this challenge is dramatically amplified due to the need to handle the network topology across the boundaries of computational domains. The second challenge is a natural tendency of dislocation lines to cluster in space (owing to the long-range interactions among the lines) and develop highly heterogeneous distributions of degrees of freedom making it difficult to achieve a good load balance. The code ParaDiS recently written at LLNL has shown much promise in addressing these challenges [15].

DD simulations of radiation-damaged materials share the challenges of fidelity and computability and add some more. As an example, the need to account for explicit dislocationSFT/loop/cluster interactions places even higher demands on the computational throughput of the DD codes. Still more complex and computationally challenging will be to describe, within a single model, the concurrent evolution of dislocation-, alloy- and radiation defect-microstructures which is a key issue for predictive modeling of fission and fusion reactor materials. Several attempts have been made to couple KMC lattice simulations of the radiation defect microstructure with the DD simulations. While possible in principle, such coupling makes both DD and KMC simulations still more expensive than 
they are in isolation. At the same time, the phase field models that have been successfully used in the past for modeling alloy microstructure evolution were recently advanced to model dislocations and their motion. However, the phase-field DD models cannot compete with the line-tracking DD when it comes to combining high resolution with computational efficiency. As a possible way to reduce the computational complexity while achieving a degree of coupling between dislocation and defect subsystems, it appears possible and in fact desirable to combine the line-tracking DD method (to handle the dislocation sub-structure) with a mesh-based phase-field approach (to handle the alloy field evolution).

\subsubsection{Atomic-level simulation of dislocations}

Computer modeling of dislocation processes relevant to damage microstructures can readily simulate several millions of atoms. With suitably designed periodic boundary conditions, dislocation glide over distances many tens of $\mathrm{nm}$ can be studied, and interaction with obstacles of realistic spacing can be treated. The importance of understanding dislocation-obstacle interaction at the atomic level was demonstrated by Rodney and Martin [16] for the glide of an edge dislocation through an interstitial dislocation loop in $\mathrm{Ni}$. The absorption of the loop and jog formation, leading to pinning effects on the dislocation, was shown in detail. Since then, simulations of dislocation interactions with SFTs, loops, voids and precipitates have been reported, e.g. [2,17,18], including the screw dislocation [19]. With suitable boundary conditions for application of force or displacement, it is possible to extract quantitative information on the relationships between applied stress, strain rate, temperature, obstacle size and spacing for a given material [2,18]. However, with limited MD timescales ( ns), plastic strain rates of at least $10^{6} \mathrm{~s}^{-1}$ are unavoidable. Thus, results on, say, applied stress and/or dislocation configuration have to be tested against changes in strain rate and temperature in order to assess their possible applicability to much longer timescales.

As an alternative to MD, molecular statics (MS) may be employed to mimic the equilibrium state at $0 \mathrm{~K}$ by minimizing the potential energy of the system. With many atoms and complex reactions, it is not always possible to guarantee that a true minimum is achieved, and processes that depend on temperature may be missed. Nevertheless, by increasing the applied strain incrementally, equilibrium line and obstacle configuration under increasing stress can be found, e.g. [2,18]. This approach has the advantage that the results should be directly comparable with those given by models based on elasticity theory for the equilibrium of dislocations under stress. In fact, MS simulation of strengthening due to voids and precipitates in $\mathrm{Fe}[2,18]$ show that the earlier continuum treatments of these obstacles $[2,20]$ have wider applicability than expected. The next challenge is to extract the effective obstacle shear resistance stress from the MS and/or MD simulations and incorporate them in DD simulations, thereby achieving nano-scale accuracy in micro-scale modeling.

\subsection{Coupling Schemes}

\subsubsection{Empirical force field $\leftrightarrow$ continuum (MM/CM)}

The way different zones exchange information is crucial for the performance of a hybrid scheme. Several schemes have been suggested for the coupling between atoms in the empirical force field zone and the nodes of the numerically discretized continuum [21-24]. Ideally one would like to meet two requirements: (i) energy conservation and (ii) bias-free forces in the boundary area. However, a critical review of currently available methods [25] clearly reveals that one cannot achieve both. The schemes of $[21,22]$ fulfill criterion (i) but require additional ghost forces to keep the boundary atoms on their lattice sites. The other schemes [5,22] neglect the concept of a global Hamilton function with the benefit of an exact representation of boundary atom forces. Since the motion of nodes is conceptually not intended to represent the thermal energy of the continuum, one might think that requirement (i) is less important. Additional complications occur when short wave length disturbances cross the zone boundary. 
Differences in the high frequency part of the phonon dispersion laws of the different zones cause backscattering of the short-wavelength part of the transmitted elastic wave. This artifact can by avoided by applying a low-pass filter at the boundary absorbing the high-frequency components [22].

\subsubsection{Quantum force field $\leftrightarrow$ empirical force field (QM/MM)}

In addition to the exchange of atomic displacement information at the zone boundary, the electrons in the quantum zone require electronic information from the MM region (which is at first not available). According to Kohn's principle of near sightedness [26] only the electrons in a small boundary layer of the MM region influence the solution of the Schrödinger equation in the QM zone. Depending on the material, the problem to obtain electronic information in the MM zone can be solved in different ways. Ionic systems represent the simplest material class, since only the Madelung potential from the MM region influences the QM solution. For covalent systems an artificial hydrogen saturation of the bonds crossing the QM/MM boundary can be performed [27]. An alternative Green's function (G) approach assumes the presence of the lattice G outside the QM region [28] as a QM boundary condition. For metallic systems, a bond order expansion of the electronic density of states leads to a real space method [29] requiring only atomic information from the MM medium.

\subsubsection{Learn on the fly}

The so called learn on the fly method (LOF) represents an alternative to the traditional QM/MM coupling [30]. The whole QM/MM region is described by a suitable empirical force field with spatially varying parameters. The force field is locally reparameterized (using a corresponding quantum force calculation for a localized cluster of atoms) if strong deviations from equilibrium configurations occur during the dynamical evolution of the system.

\subsubsection{Related questions and future development}

The most important question is the relevance of simultaneous-multiscale schemes for fusion and Gen-IV fission related materials. The MD description of radiation damage and related component failure represents an important cornerstone on the way to an understanding and design of the required strong materials. In the past, simulations were focused on a qualitative understanding of the relevant processes and it was sufficient to employ simple empirical potentials for a very limited class of materials (Lennard-Jones $\mathrm{Ar}$ and embedded atom $\mathrm{Cu}$ are typical examples) and using very simple boundary conditions (fixed atoms, periodic boundary conditions, analytic asymptotic continuum solutions). This clearly marks the childhood of computational materials science. In order to enter maturity more elaborate models with quantitative predictive power are required. Alloys are by far more complicated than pure copper and therefore the accuracy of the interatomic forces is a pressing problem. For localized disturbances, QM/MM or LOF schemes might provide valuable tools to achieve high precision where it is needed. The success of these methods will also depend on the development and improvement of linear scaling electronic structure methods which will allow accurate treatment of ten thousands of atoms in the near future. For a quantitative description of even larger non-equilibrium systems, empirical potentials with near quantum precision are mandatory and development into this direction also needs a strong support. Empirical bond order alloy potentials are a significant step in this direction [31].

In order to be really predictive, one must not neglect the exact influence of macroscopic boundary conditions on the outcome of the microscopic dynamics in high energy collision cascades, thermal spikes and crack motion. For crack propagation [32] or energetic particle surface collisions [33] it is already a standard procedure to embed the atomistic region into a continuum. For radiation damage of $\mathrm{MeV}$ cascades, it has to be applied in the near future [34].

Up to now the continuum is treated with the discretized differential equations of elasticity theory. Other continuum descriptions using mesoparticle dynamics [35] would allow for crack 
propagation from the microscopic zone into the embedding medium. The development into this direction is very interesting and could result in a powerful alternative to span the length scales.

\subsection{Multiscale Modeling of Thermodynamics and Kinetics under Irradiation}

A first step in the modeling of microstructure evolution is to predict the thermodynamic properties of complex systems, not always available experimentally. In addition to first principles calculations of cohesive energies, from which formation enthalpies [36] are deduced, very powerful numerical calorimetry methods based on Static MC methods have been developed which give access to the thermodynamic functions of complex systems where the configuration entropy cannot be calculated explicitly [37]. This is the case of amorphous or strongly distorted crystalline structures.

The Rigid Lattice KMC methods are extremely efficient for studying the incubation and nucleation of precipitates, and can now be validated with experiments at the same scale as the modeling box $\left(10^{-5} \mu \mathrm{m}^{3}\right)$ using the $3 \mathrm{D}$ Atom Probe, a technique which has recently achieved close to atomic resolution. They are used to identify the complex sequence of metastable and stable precipitation (or "dynamic phase diagrams") in alloys, to control materials strength and brittleness by influencing nucleation and coarsening, notably by identifying additives to enhance nucleation and hinder coarsening. They are particularly well adapted to test and integrate refined energetic and diffusion models, e.g. [38]. Recently, they have been upgraded to tackle irradiation effects by implementing all the interstitial diffusion mechanisms, PD sources and sinks, thus allowing for point defects fluxes towards sinks which are responsible for Radiation-Induced Segregation and Precipitation (RIS \& RIP). As they give access to the details of evolution at the atomic scale for all the atoms in the calculation box, these techniques are demanding in CPU time (roughly proportional to the number of jumps performed) and therefore limited to relatively small time and space scales, which in turn depend on temperature and atomic binding energies that control the jump probabilities. Also, they require knowledge of elementary parameters (binding and saddle point energies), which must be very accurate in view of the high sensitivity of kinetics to some of these parameters, whose number, in addition, becomes quite large in even moderately complex alloys. The massive input of first principles data is therefore crucial.

A limitation of the Rigid Lattice approach is that it does not allow for systems and phenomena with large size effects (i.e. large elastic constraints), at least not with an accuracy equivalent to that achieved for the effects of purely chemical bonds. For such situations, however, Relaxed Lattice MC techniques are being developed to account for diffusion and precipitation in systems with large atomic and/or particle size effects, notably the coherent-incoherent transition in precipitation. These developments are promising, but are hindered by the enormous CPU time requirement [39].

Systems of larger dimension $\left(\mu \mathrm{m}^{3}\right)$ and comprising complex objects can be explored with the help of Event-Based KMC models, in which the MC algorithm is not applied to individual atomic jumps but to the probability of impingement or dissociation of populations of mobile defects and immobile objects [40]. This is possible at the expense of detailed spatial localization, which is no longer at the atomic scale. Such models are well adapted to account for recovery of complex point defects and defect cluster populations in pure metals and dilute alloys during and after irradiation [41], and especially the effect of microstructural features such as dislocations, grain boundaries, precipitates, free surfaces etc, which of course requires that their sink and source strengths are correctly implemented.

In order to deal with concentrated, multi-component, heterogeneous systems over wider time and space scales, Self-Consistent Mean Field diffusion models are used. They describe the diffusive and thermodynamic properties at the same level of approximation, accounting for all the correlations between atomic jumps, and for both the thermodynamic and kinetic coupling of fluxes. The latter is of paramount importance for materials under irradiation as it gives rise to RIS at grain boundaries in stainless steels [42], for example. Also, such models are designed to take advantage of existing macroscopic data bases (e.g. DICTRA) and enrich them with atomic scale-based calculations. 
In order to describe the overall evolution of single defect and defect cluster populations without space or time limitation, one can resort to Rate Theory (Cluster Dynamics) in conjunction with TEM and 3D Atom Probe experiments, e.g. to understand the formation mechanism of defects and solute clusters responsible for the hardening and embrittlement of steels under neutron irradiation [43]. They have been recently extended and applied to precipitation in cubic alloys and the irradiation growth of hexagonal metal systems.

The atomistic approach is also used to check, validate and improve mesoscopic kinetic models, namely nucleation, growth and coarsening theory. This formalism is based on a few basic quantities, essentially the nucleation driving force and the interface free energy, which are not accurately known but can be calculated on a safe physical basis using first principles methods, atomic scale models of interactions and diffusion, and statistical mechanics techniques (e.g. precipitation in complex alloys [44]).

\section{References}

1. D.J. Bacon, U.F. Kocks and R.O. Scattergood, Phil. Mag. 28 (1973) 1241.

2. Yu.N. Osetsky and D.J. Bacon, Model. Simul. Mater. Sci. Eng. 11 (2003) 247.

3. J. Marian, W. Cai and V. Bulatov, Nature Materials 3 (2004) 158.

4. C.C. Fu, F. Willaime and P. Ordejon, Phys. Rev. Let. 92 (2004) 175503.

5. M. Fähnle Defect and Diffusion Forum 194-199 (2001) 279.

6. M.I. Mendelev, S. Han, D.J. Srolovitz and G.J. Ackland, Phil. Mag. 83 (2003) 3977.

7. G.J. Ackland M.I. Mendelev, D.J. Srolovitz, S. Han and A.V. Barashev, J. Phys. C, in press (2004).

8. G.J. Ackland and S.K. Reed, Phys. Rev. B 67 (2003) 174108.

9. A.F. Voter, F. Montalenti and T.C. Germann, Annual Rev. Mater. Res. 32 (2002) 321.

10. M.R. Sorensen and A.F. Voter, J. Chem. Phys. 112 (2000) 9599.

11. B.P. Uberuaga et al, Phys. Rev. Let. 92 (2004) 115505.

12. A.F. Voter, Phys. Rev. B 57 (1998) 13985.

13. H. M. Zbib, V. Bulatov and T. Diaz de la Rubia, Trans. ASME 74 (2002) 78.

14. N. M. Ghoniem, S.-H. Tong, and L. Z. Sun, Phys. Rev. B 139 (2000) 913-927.

15. V. Bulatov, W. Cai, J. Fier, M. Hiratani, T. Pierce, M. Tang, M. Rhee, K. Yates and T. Arsenlis, Supercomputing 2004, submitted.

16. D. Rodney and G. Martin, Phys. Rev. B 61 (2000) 8714.

17. J.S. Robach, I.M. Robertson, B.D. Wirth and A. Arsenlis, Philos Mag. 83 (2003) 995.

18. Yu. N. Osetsky, D. J. Bacon and V. Mohles, Phil. Mag. 83 (2003) 3623.

19. D. Rodney, Acta Mater. 52 (2004) 607.

20. R.O. Scattergood and D.J. Bacon, Acta Metall. 20 (1982) 1665.

21. S. Kohlhoff, P. Gumbsch and H.F. Fischmeister, Phil. Mag. A 6 (1991) 851.

22. E.B. Tadmor, M. Ortiz, and R. Phillips, Phil. Mag. A 73 (1996) 1529.

23. M. Moseler, J. Nordiek and H. Haberland, Phys. Rev. B 56, (1997) 15439.

24. J. Q. Broughton, F. F. Abraham, N. Bernstein, and E. Kaxiras, Phys. Rev. B 60 (1999) 2391.

25. W.A. Curtin and R.E. Miller, Model. Simul. Mater. Sci. Eng. 11 (2003) 33.

26. W. Kohn, Phys. Rev. Let. 76 (1996) 3168.

27. F.F. Abraham, J.Q. Broughton, N. Bernstein and E. Kaxiras, Europhys. Let. 44 (1998) 783.

28. N. Bernstein, Europhys. Let. 55 (2001) 52.

29. A.P. Horsfield, A.M. Bratkovsky, D.G. Pettifor and M. Aoki, Phys. Rev. B 53 (1996) 1656.

30. A. de Vita and R. Car, Materials Research Society Symposium Proceedings 491 (1998) 473.

31. K. Albe, K. Nordlund and R.S. Averback, Phys. Rev. B 65 (2002) 195124.

32. J. Riedle, P. Gumbsch and H.F. Fischmeister, Phys. Rev. Let. 76 (1996) 3594.

33. M. Moseler, O. Rattunde, J. Nordiek and H. Haberland, Nucl. Instrum. and Meth. B 164 (2000) 522.

34. K. Nordlund, private communication.

35. B.L.Holian, Europhys. Let. 64 (2003) 330. 
36. R. P. Gupta et al., Phil. Mag. A 80 (2000) 2393.

37. M. Athènes, Phys. Rev. E 66 (2003) 16701 and 46705.

38. F. Soisson et al., Phys. Rev. B 62 (2000) 203; 65 (2002) 094103.

39. J.L. Bocquet, Defect and Diffusion Forum 203-205 (2002) 81.

40. J. Dalla Torre, J.L. Bocquet, N.V. Doan, G. Martin and E. Adam, in Microstructural Processes in Irradiated Materials, TMS Symposium, San Diego, March 2003.

41. C.C. Fu, J. Dalla Torre, F. Willaime, J.L. Bocquet and A. Barbu, to be published

42. M. Nastar, Defect and Diffusion Forum 194-199 (2001) 171; and in Microstructural Processes in Irradiated Materials, TMS Symposium, San Diego, March 2003; Phil. Mag. A, in press.

43. F. Christien and A. Barbu, J. Nucl. Mater. 324 (2003) 90.

44. E. Clouet, M. Nastar and C. Sigli, Phys. Rev. B 65 (2002) 94105. 


\section{Session III: Goals and Targets for Understanding and Predicting Material Performance}

\subsection{Objectives of Session III}

The primary objective of this session was to define the goals and targets required for modeling and simulation research to develop a predictive capability for material performance under the irradiation conditions of fusion and Gen-IV fission reactors. Two main aspects of defining these objectives became clear:

(1) identifying the critical problems for structural materials in the irradiation environment, and clearly describing the required development pathway, and

(2) identifying the limitations of present day computational and modeling capabilities with respect to reaching the goals and objectives defined above, while pointing out possible approaches that can lead to achievement of the stated goals.

\subsection{Background and Primary Structural Materials Issues}

Development of materials that can perform reliably for long times ( $\sim 60$ years) in the various Gen-IV and fusion reactor environments is a substantial challenge. In particular, fusion structures will place unprecedented demands on materials and complex engineering systems. The demands are driven by time-varying thermal-mechanical loads on large heat transfer structures with complex interconnected geometries. At the same time, structural materials will face in-service degradation of their performance sustaining mechanical properties by suffering internal damage development, macroscopic cracking, corrosion, and inherent dimensional instabilities [1,2]. The result will be numerous and hard to predict failure paths in the face of demanding requirements for high reliability, long life and demonstrable safety margins. It is critical to not only develop advanced high performance materials and models for predicting their in-service changes, but also methods to interpret these property changes in the context of engineering assessments of integrity limits in actual reactor structures.

Structural materials are characterized by complex, multi-constituent, multiphase, highly defected, non-equilibrium microstructures that mediate an array of complex mechanical properties. Properties that must be reliably modeled and predicted include:

- Yield strength and strain hardening constitutive laws.

- Various types of 'ductility'.

- Fatigue crack growth rates.

- Fracture toughness.

- Irradiation creep rates.

- Thermal creep rates.

- Void swelling rates.

- Creep rupture times and strains.

- Thermo-mechanical fatigue stress and strain limits.

- Creep crack growth rates.

- Creep-fatigue interactions.

- Environmentally assisted cracking.

- Bulk corrosion, oxidation and compatibility.

The governing processes involve many degrees of freedom, are inherently multiscale (time/length), and are controlled by multiple physical mechanisms that act synergistically. Critical 
outcomes (e.g., void swelling) often depend on small differences between large competing effects. In addition, material properties and failure paths are often influenced by extrinsic factors on the scale of both individual test specimens and engineering structures. Thus, a primary objective must be to develop physically-based property models that account for the highly synergistic combinations of the relevant material and environmental variables. These variables include:

- The irradiation temperature $\left(\sim 250\right.$ to more than $\left.800^{\circ} \mathrm{C}\right)$

- The atomic displacement rate (dpa/s) and the total dose in displacements per atom (50 to $200 \mathrm{dpa}$ )

- Neutron energy spectrum, which determines the PKA spectrum and cascade size

- Total helium and hydrogen generation (500 to 2000 and 2000 to 8000 appm, respectively)

- Other transmutations reactions (burn-in and burn-out of essentially all elements)

- Stress and stress-state (very high thermal plus primary stresses)

- The cyclic time dependence of imposed stresses and strains

- The evolutionary history of the environmental variables listed above

- The alloy type, composition and processing-fabrication history (including impurities and the complete start-of life microstructure)

- Numerous extrinsic factors related to the size, geometry, stress-state, temperature, loading rate that are imposed on the specimen or structure, potentially leading to failure

Given that the influence of one variable generally depends on the combination of nearly all others, and the fact that properties evolve in a history-dependent manner rather than being simply controlled by state-variables, purely empirical approaches to predictions are prohibited by the large number of variable states. Hence, physically-based modeling is mandatory. However, the property models must be verified by high quality databases to provide a reliable foundation for interpolation and extrapolation and to quantify scatter and uncertainties. Given the complexity of the challenge, this will require iterative cycles of modeling and various kinds of experiments to build both physical understanding and a knowledge base [2].

\subsection{Cross-Cutting Challenges for Materials Development}

Fortunately, many of the challenges cut across various technologies and materials. Further, models of microstructural evolution can form a common basis for many properties. Models can most often be hierarchical and physics-based, linking atomic to structural scales. Useful knowledge and models can be developed without perfect theory. This approach has been used to develop robust hierarchical, physically-based models of reactor pressure vessel embrittlement $[3,4]$ and other important radiation damage effects in current light water reactor components. In many cases, the models provided predictions of important, but unexpected, phenomena that were only subsequently verified by experiment.

This experience is particularly pertinent to the leading candidate alloys for fusion and many Gen-IV fission applications. These include both low-activation normalized and tempered martensitic steels (LAMS) used in the range of 300 to $550^{\circ} \mathrm{C}$ (note higher temperatures have been proposed for Gen-IV applications, but this seems problematic) and advanced high temperature nanostructured ferritic alloys (NFA) strengthened by a very large population of nm-scale Y-Ti-O enriched precipitates [5,6]. As a result of the broad interest in LAMS and NFA, it is recommended that modeling studies primarily focus on these material systems, which at least to some extent reduces the number of important material variables. 


\subsubsection{Deformation and fracture}

Deformation and fracture behavior is a primary example of a cross-cutting materials performance phenomena. Depending on the specific material, a range of mechanisms can interact render the structural material brittle at either low or high temperatures. The interplay between these mechanisms is complex resulting in deformation and fracture properties that depend on many intrinsic and extrinsic variables. As a result, any successful model must account for a number of environmental variables (temperature, dose, dose rate, $\mathrm{He}$ and $\mathrm{H}$ levels, stress, etc.), and these same variables need to be considered in the development material property databases for model validation. A successful model must be hierarchical, incorporating information from the atomistic through mesoscale microstructural evolution leading to the prediction of constitutive relationships and macroscopic fracture.

The main cross-cutting issues in deformation and fracture are listed below. These are discussed in somewhat more detail for the LAMS and NFA in section 3.5.

- Irradiation effects on stress-strain, constitutive laws, and consequences of flow localization;

- Validity and physical basis of the Master Curve (MC) for predicting the ductile-to-brittletransition;

- Embrittlement - MC shifts due to hardening and He effects;

- Model-based designs for high performance alloys;

- Irradiation effects on constitutive properties: $J_{2}$ laws linked to microstructure evolution;

- Development of plasticity models for constitutive properties, for example bridging between dislocation dynamics, crystal plasticity, and polycrystalline plasticity;

- Understanding flow localization and ductility loss of irradiated materials;

- The apparent universality of the MC shape, and the physical basis for this universality;

- Effects of helium on grain boundaries, and how that influences shifts in DBTT; and

- Model-based design of alloys, for example, including a high density of nano-clusters to trap helium in high-pressure bubbles and thus preventing them from going to grain boundaries.

Sophisticated multiscale modes must be developed to make progress in understanding these issues, and to develop strategies for mitigating the effects of radiation on deformation and fracture properties. Models that are firmly based in understanding the underlying physical phenomena are imperative. Advances in computational tools will be required make the implementation and use of these more complex models feasible.

\subsubsection{Helium and hydrogen effects}

Helium is produced in essentially all the structural materials of interest under neutron irradiation via $(n, \alpha)$ reactions. As discussed above, the relative contribution of thermal and fast neutrons varies among different materials and irradiation environments, but total helium generation in steels is about 1 appm/dpa in a fast fission spectrum and 10 to $20 \mathrm{appm} / \mathrm{dpa}$ under DT fusion reactor conditions. Several methods have been employed for modeling helium effects in irradiated materials during the past two decades. An early simulation using a rate theory model indicated that void swelling may not be a monotonic function of He/dpa ratio as shown in Figure 3-1 [7]. A peak in swelling is observed near the fusion-relevant value. The data shown in Figure 3-1 were obtained from fission reactor irradiation of stainless steel in both fast (FFTF) and mixed spectrum (HFIR) reactors. These produce He/dpa ratios much lower and higher than DT fusion, respectively. The fusion relevant value was obtained in the Oak 
Ridge Research Reactor (ORR) by manipulating the neutron energy spectrum. Similar non-monotonic behavior was also observed in dual ion irradiation experiments in which helium was implanted at different rates while heavy ions (e.g. Fe, Ni) were used to simultaneously create displacement damage [8].

Figure 3-1. Comparison of model predictions and fission reactor irradiation data on influence of $\mathrm{He} / \mathrm{dpa}$ ratio on void swelling.

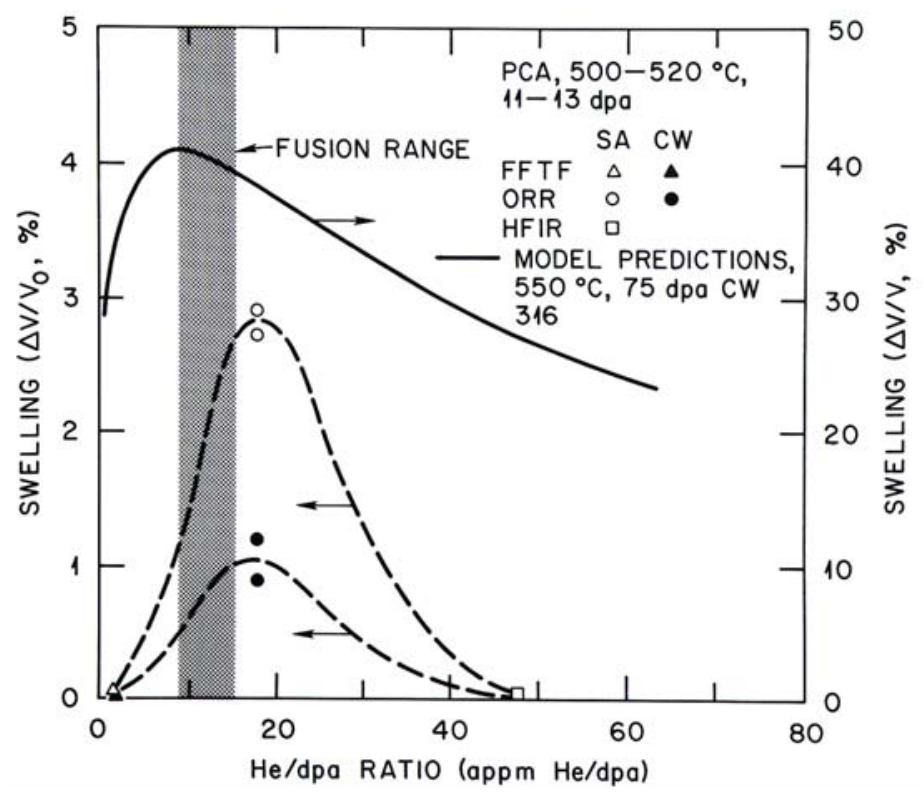

Atomistic simulations employing molecular dynamics are now being used more extensively to determine the energetics of binding and migration of various helium-point defect complexes. Additional work is needed to verify the results obtained with empirical potentials that do not yet fully account for potential interactions of $\mathrm{He}$ with the host lattice. Such atomic level information is passed on to mesoscale simulations of microstructure evolution based on reaction rate theory or KMC. The rate theory models generally assume that the microstructure can be described as a spatially homogeneous effective medium with discrete sinks for point defects and solutes (including He and H). The KMC models provide greater spatial detail with a concomitant increase in computational burden.

One of the key advantages of rate theory is that the results of simulations can be directly compared to experiments, where the relevant microstructural parameters are obtained from either experiments (typically transmission electron microscopy) or atomistic simulations. For example, KMC simulations can now be used to solve complex point defect diffusion problems in the stress field of dislocations, and thus derive more realistic values for the dislocation bias factors. At the same time, large systems of equations describing the nucleation and growth of void and bubble populations can be solved with current day large-scale computers, thus providing more accurate descriptions of nucleation and growth. This level of detailed rate theory modeling is essential, because experiments show that several phenomena are influenced by helium in a complex fashion. For example, as mentioned above, the swelling at any given dose is not a monotonic function of the helium-to-dpa ratio. Likewise, the effects of small helium concentrations on grain boundary fracture depend on many details of the microstructure, while the effects of helium bubbles on hardening or embrittlement at low temperature are not yet clear.

Previous work has focused primarily on the effects of helium, but further investigations with both the atomistic and kinetic models need to be directed toward the effects of hydrogen on the microstructure. Recent experimental results indicate that hydrogen may prove to have a greater than anticipated effect on microstructural evolution. For example, triple ion beam $(\mathrm{Fe}, \mathrm{He}$, and $\mathrm{H}$ ) irradiation experiments carried out at ORNL indicated that materials undergo increased hardening when hydrogen is co-implanted with helium $[9,10]$. This leads to flow localization, and substantial retention of hydrogen. A systematic understanding of these synergistic effects is still lacking. The hydrogen 
retention issue may have safety and operational significance if a higher than expected tritium inventory accumulates in fusion first wall and blanket materials.

\subsubsection{Stress corrosion cracking}

The phenomenon of irradiation assisted stress corrosion cracking (IASCC) is becoming a major problem for the operation of light water reactors. EPRI studies estimated that savings of $\$ 1$ to $1.5 \mathrm{M}$ per plant per year can be achieved if the problem of IASCC is alleviated. High radiation levels in a reactor core produce changes in iron- and nickel-base austenitic stainless alloys leading to extensive hardening, a reduction in uniform ductility, and an increased susceptibility to intergranular stress corrosion cracking (SCC). This cracking process, IASCC, is a serious concern for both boiling water reactors (BWRs) and pressurized water reactors (PWRs). Stainless steel components can become susceptible to cracking at doses less that $10 \%$ of the expected end-of-life dose and the likelihood of failure may increase with increasing service exposure. Susceptibility to IASCC is clearly linked to radiation-induced changes in the alloy microstructure and microchemistry, but fundamental understanding of controlling mechanisms has been elusive.

Experimental observations show that the fraction of Intergranular (IGSCC) versus dose shows a transition curve, and that this behavior is associated with the segregation of $\mathrm{Cr}$ to boundaries as a function of dose. Cr was also shown to diffuse away from grain boundaries, giving wide range of scatter in the $\mathrm{Cr}$ concentration. The large scatter points out to the need for more focused experiments that are based on detailed modeling assessments.

\subsubsection{Radiation stability of alloys}

Engineering alloys are typically incorporate several major and minor alloying elements among their components. While the transport and fate of minor elements under irradiation can in principle be handled with the same tools as point defects (i.e. book keeping of the mean or local concentration as a function of time), such is not the case for major alloy components or for minor elements that strongly participate in the formation of second phases. In particular, the cluster dynamics technique employed in the rate theory fails because of percolation problems.

A small community (e.g. G. Martin and coworkers at the CEA, Saclay, France; P. Bellon at the University of IL; V. G. Vaks at Kurchatov Institute, Russia; and F. Haider, Universität Augsburg, Germany) works at developing a theoretical framework to assess the stability of stationary phases under irradiation. At the present time, it is known that phase stability simultaneously depends on the temperature, composition, neutron dose and dose rate, and the PKA energy (cascade size). This implies that both the spatial extent of the cascade and the number of replacements per cascade are important factors. For the overall approach to be successful, the evolution of the precipitate population under irradiation must be simultaneously evaluated along with the defect sink structure (dislocation network, defect aggregates of various forms). These two components evolve at much different rates. The microstructural sinks evolve at a rate proportional to the small difference between two relatively large quantities, the vacancy and the interstitial fluxes to sinks. However, the precipitates grow or shrink because of the coupling of the solute flux with both of these two point defect fluxes, an additive process $[11,12]$.

\subsection{Required Research and Development}

Models that are to be developed must be hierarchical, predicting macroscopic properties based on knowledge of processes starting from the atomic information. An important task is to design the architecture for these models, and to efficiently define their connections. One successful example of this approach is modeling of pressure vessels, where related multiscale models and experiments have been integrated to predict the embrittlement of steels. However, most of that effort relied on manually passing information between models that describe different length and time scales. A more integrated approach 
is being adopted in current research in the European Union. Three prominent areas requiring further model development and integration are described in the following sections.

\subsubsection{Interatomic potentials for radiation damage}

There are a large number of published interatomic potentials designed for various purposes, whose suitability for use in radiation damage studies varies. The crucial properties for radiation damage simulations are:

(1) Point defect formation, migration and interaction energies;

(2) Elastic constant anisotropy;

(3) Grain boundary energetics;

(4) Dislocation structure and response to stress; and

(5) Alloy phase stability.

None of these properties are necessarily predicted correctly because of the incomplete physical basis of the potentials. With pair-wise interactions, some properties are necessarily wrong. With many-body potentials (used here as a generic term covering glue, Finnis-Sinclair, embedded atom, modified embedded atom, and effective medium theory potentials), many properties can be fitted provided "correct" values are available. These types of potentials have been the "state of the art" for 20 years.

Historically, there has been an insufficient database for robust fitting, parameterization, and verification of potentials. In many cases, the desired data can not be obtained experimentally. Recent renewed interest in interatomic potentials is based on the ability of $a b$ initio calculations to provide this missing data and, with teraflop machines, verification of many predictions is finally possible. The ability to carry out $a b$ initio calculations in systems of up to a few hundred atoms has allowed more accurate verification procedures to be applied to existing models, and also highlighted some serious flaws. Where tested against new data, existing potentials have generally proved disappointing. Some common problems include poor interstitial formation energies, too small energy differences between alternate configurations, and the lack of a satisfactory description of the austenitic-ferritic transition in iron. Some of these problems can be traced to problems in parameterization of the potentials; but others, such as the absence of a physically sensible treatment of magnetization, point to more fundamental problems in the many-body potential concept.

There are reasonably good prospects for rectifying many of these problems; some simply require reparameterizing existing models and extending them to systems where previously insufficient underpinning data existed. Others require implementation of new models which have already been proposed, and some may require the development of new approaches. The primary resource requirement for the development of the required potential models is manpower, inspiration and stronger interactions between research groups to understand one another's needs.

The majority of the effort in potentials for metallic phases has focused on elemental materials. Potentials for multi-component systems have been developed in isolated cases, but the predictive capability of these potentials is typically disappointing. Reasonable models for the mission-critical helium impurities exist or are under development. There are at least two challenges in the development of potentials in alloy systems. First, there is generally much less fundamental data available, although this can be rectified through the use of $a b$ initio calculations. Second, the appropriate functional forms are not as well developed. Most potentials are based on simple pictures of bonding. In alloy systems, the nature of the bonding is inherently more complex suggesting that more sophisticated potentials are needed to describe the energetics reliably. Non-metallic impurities (carbon, phosphorus) are even more problematic. More details on recent and future development efforts in the area of interatomic potential is provided in the report from Session II. 


\subsubsection{Dislocation interactions and dynamics}

One of the critical problems for the development of radiation-resistant structural materials is the embrittlement arising from a loss of ductility and plastic flow localization [13,14]. Modeling the interaction between dislocations and radiation-induced obstacles is providing great insights into the physics of this problem, and will eventually lead to the design of radiation-resistant structural alloys.

Models of dislocation-defect interactions are pursued on two levels: (1) the atomistic level, where MD simulations are playing significant role; and (2) the mesoscopic level, where DD simulations are providing insights into larger-scale behavior. Both types of models are complementary, and provide direct information for experimental validation on the effects of irradiation on hardening, yield drop, and plastic flow localization, etc. Atomic scale models are used to inform DD models on the details of dislocation-defect interactions. Presently, MD models can simulate 1 to 10 million atoms on a routine basis [15]. Both static and dynamic simulations are used. For static simulations, fixed displacement boundary conditions are applied, and conjugate gradient minimization is used. Newtonian equations of motion are used for dynamic simulations, and either force or velocity conditions are applied on boundary atoms. Atomistic simulations have shown the range where elasticity estimates are valid for dislocation-defect interactions, and where they break down due to new mechanisms. For example, the interaction of dislocations with small precipitates can result in local phase transitions and an associated energy cost that cannot be predicted from DD models. Also, it has been shown that dislocation-void interaction leads to dislocation climb, and the formation of a dislocation dipole before the dislocation completes cutting through the void completely. These effects are all of an atomic nature, and the information should be passed on to DD simulations.

A number of challenges remain in the area of dislocation-defect interactions, as described in the following list:

(1) The strain rates in MD simulations are far in excess of experimentally achievable rates, and methods to incorporate slow rate events due to temperature or force field fluctuations have not yet been developed.

(2) The information passing between MD and DD is not systematic yet. For example, the "angle" between dislocation arms before it leaves the obstacle is often used in DD simulations as a measure of obstacle strength. However, the definition of this angle in both MD and experiments is problematic for a variety of reasons. Force-displacement information will be necessary.

(3) Methods for incorporating lower length scale microstructure effects into DD simulations are not well developed. For example, we do not have information on obstacle dynamics, solute effects, dislocations near cracks, dislocation nucleation, etc.

(4) The size of atomistic simulations is very small, and cannot deal with complex dislocation structures. Methods for reducing the degrees of freedom are needed.

(5) The boundary conditions used in MD simulations are either periodic, fixed, or represented by elastic Green's functions. General methods for embedding MD simulations into the continuum are in an early stage of development.

(6) DD codes are limited to small size crystals. To improve their speed and range of applicability, new methods of designing these codes on massively parallel computers are needed.

(7) The connection between DD and macroscopic plasticity has not yet been made through "coarse graining" and a systematic reduction of the degrees of freedom. Development of this area is essential to the prediction of constitutive relations and macroscopic plastic deformation. 


\subsubsection{Alloy stability}

The strategy for future improvement in modeling the stability of multiphase alloys under irradiation requires considerable theoretical progress and much computational work. One approach to assessing the stability of a given phase under irradiation is to study the growth and/or decay rate of a model precipitate in a model solid solution under irradiation. Phase field models [12] provide the appropriate tool, provided essential progress is made to implement atomistic mobility coefficients in these models. Indeed, phase stability under irradiation is affected by the details of the coupling of solute and defect fluxes, a feature not yet taken into account by phase field models. A second issue is determining how to account for the temporal and spatial correlation inherent in the defect source which arises from discrete atomic displacement cascades. Improving the theory of noise terms in phase field models is one route in this direction.

Once progress has been achieved in developing the basic phase field models, the computational burden of the simulations will be significant. Obtaining results will involve the solution of coupled PDEs on a large grid, with systematic variations in key parameters evaluated (point by point construction of phase boundaries in the phase diagram while varying composition, temperature, dose rate, cascade size, etc.). International cooperation should be used as an avenue to more rapid progress in this area.

\subsection{Challenges to Alloy Performance: Candidate and Developmental Alloys}

Two specific alloy systems will be used as examples to illustrate the complexity of the phenomena that the anticipated modeling effort must address, and to demonstrate a potentially fruitful development path. These are the current prime candidate alloy for nearer-term applications, the lowactivation martensitic steels (LAMS) and the developmental ferritic alloys that are strengthened with a dispersion of nanometer-scale oxide particles, the so-called nanostructured ferritic alloys (NFA).

\subsubsection{Performance of current materials: LAMS}

LAMS typically contain 8 to $9 \% \mathrm{Cr}$ and 1 to $2 \% \mathrm{~W}$ as the primary alloying elements, along with about $0.1 \% \mathrm{C}$ and smaller quantities of carbide forming micro-alloying elements like Mo, $\mathrm{V}$, and Ta as well as small amounts of $\mathrm{Mn}$ and $\mathrm{Si}$. Major phases include a variety of alloyed carbides and, depending on the irradiation temperature and dose, $\mathrm{Cr}$-rich $\alpha^{\prime}$ and $\mathrm{Fe}_{2} \mathrm{~W}$ Laves phases [16]. The microstructures are composed of dislocation and dislocation substructures inside martensitic laths, forming small groups of lath packets within the prior austenitic grains. These coarse scale $(>0.05 \mu \mathrm{m})$ structures and phases form during processing and are generally stable at low-to-intermediate irradiation temperatures. In the regime below about $400^{\circ} \mathrm{C}$, the dominant features induced by irradiation are dislocations loops, gas bubbles and voids, and in some cases, fine scale precipitates like $\alpha^{\prime}$. These fine-scale features cause hardening, loss of uniform tensile strain capacity, flow localization and embrittlement. The effects of hardening may be amplified by high levels of $\mathrm{H}$ and $\mathrm{He}$ if sufficient quantities lead to grain boundary decohesion and a brittle intergranular fracture mode up to very high temperatures. Irradiation creep occurs over the entire range of service temperatures and is the dominant source of dimensional instability at low-to-intermediate temperatures.

The sizes and number densities of the features decrease with increasing irradiation temperature. In the range of 400 to $500^{\circ} \mathrm{C}$ high He levels may result in enhanced swelling associated with the biasdriven vacancy flux transforming stably growing bubbles to unstably growing voids. Above about $450^{\circ} \mathrm{C}$ (or perhaps a lower limit lower with increasing dose) the coarser microstructures and phases become increasingly unstable and tend to recover and coarsen, while precipitation of grain boundary Laves phases occurs. This evolution can lead to both softening and non-hardening embrittlement. Irradiation and applied stresses may accelerate and lower the temperature range of the time-temperature C-curves describing these transformations by a variety of mechanisms, including radiation enhanced diffusion. However, experimental observations do not generally show large irradiation 'driven' effects 
on microstructural stability, or phenomena such as severe solute segregation in LAMS. The main concern at temperatures above $500^{\circ} \mathrm{C}$ is the accumulation of $\mathrm{He}$ on grain boundaries. This may be accompanied by severe reductions in creep rupture times and strains due to stress driven growth of creep cavities that nucleate on bubbles. The high sink density in LAMS is believed to offer some degree of grain boundary protection, but this has not been verified under fusion relevant conditions. Indeed, a major challenge to predicting the performance of materials in fusion environments is the absence of a high-energy, high-dose neutron source. However, fission reactors can be used to study the effects of high levels of helium by combinations of spectral tailoring and doping with isotopes of elements with high and low $(n, \alpha)$ cross sections (B and Ni) and more recently, in situ $\alpha$-implantation of LAMS (and virtually any material) from thin adjoining layers rich in these elements. Information from these experiments will be used to develop, calibrate and validate multi-scale models of the effects of damage accumulations, including the transport and fate and consequences of high levels of helium.

Progress on developing engineering property models for these alloys for fusion and advanced fission and accelerator-based applications will require a large long-term well-organized, international effort. Some very high priority and potentially feasibility limiting cross-cutting issues must be addressed in the near to intermediate term. These include:

- Irradiation hardening effects on true-stress strain constitutive laws, the causes and the consequences of flow localization and their combined implications to effective 'ductility'.

- Validity \& physical basis for the Master Curve (MC)-Shifts $\left(\Delta \mathrm{T}_{\mathrm{o}}\right)$ method for measuring/applying fracture toughness in the cleavage transition, i.e. verify the apparent universal fracture toughness temperature MC shape; determine size and geometry effects on effective fracture toughness; and characterize large increases in transition temperature shifts $\left(\Delta \mathrm{T}_{\mathrm{o}}\right)$ from synergistic hardening and non-hardening embrittlement mechanisms, including helium and hydrogen effects.

- Irradiation embrittlement in the ductile tearing and intergranular fracture regimes.

- High temperature creep/creep rupture and ductility, including helium effects.

- Dimensional instabilities due to possible void swelling and particularly irradiation creep, including helium and hydrogen effects.

- Model based design of high performance radiation resistant alloys for managing displacement and transmutant gas damage.

- Complex models of time-dependent structural loading/deformation based on advanced constitutive-failure - models and integrity assessment methods

- Development of processing, manufacturing and joining models for high performance, high temperature materials (alloys, ceramics and composites) with a good balance of properties.

Other important, but more complex properties, such as thermal-mechanical fatigue, creep crack growth and environmentally assisted cracking, will require a longer-term effort. It is beyond the scope of this summary to discuss these in more detail. However, one example of a problem that must be solved for fusion materials and a possible approach to solving it will be presented. This is the damaging effects of helium and hydrogen on embrittlement in the LAMS. These gases are produced at a rate of about 10 and $40 \mathrm{appm} / \mathrm{dpa}$, respectively, irradiation to $100 \mathrm{dpa}$ will generate on the order of 1000 appm helium and 4000 appm hydrogen. Both of these transmutation products individually, and in synergistic combination with each other and displacement damage, result in degradation of a wide variety of properties, such as creep rupture time and ductility at higher service temperatures. High concentrations of helium and hydrogen may also significantly worsen irradiation embrittlement in LAMS due to 
displacement damage induced hardening at low to intermediate temperatures. We now focus on the latter issue to illustrate the challenges.

Both experiments and micromechanical models show irradiation hardening, or increases in the yield stress $\left(\Delta \sigma_{\mathrm{y}}\right)$, produce upward shifts in the master toughness-temperature curves $\left(\Delta \mathrm{T}_{\mathrm{o}}\right)$ at a rate of about $0.6^{\circ} \mathrm{C} / \mathrm{MPa}$ [17]. Since peak hardening is expected to be on the order of 500 to $600 \mathrm{MPa}$ for irradiations at lower to intermediate temperatures, large $\Delta \mathrm{T}_{\mathrm{o}}$ of more that $300^{\circ} \mathrm{C}$, corresponding to inservice $\mathrm{T}_{\mathrm{o}}$ of $200^{\circ} \mathrm{C}$ or more are possible from displacement damage alone. However, recently spallation proton data has shown that bulk helium levels of 600-800 appm also severely weaken grain boundaries. Reduced local fracture stresses interact synergistically with large amounts of irradiation hardening, producing very brittle intergranular (IG) facture. Estimates of combined effects suggest $\mathrm{T}_{\mathrm{o}}$ up to $500^{\circ} \mathrm{C}$ may be possible [17,18]. This is clearly a basic feasibility issue for use of LAMS in fusion structures. High concentrations of hydrogen may also be very damaging $[9,10]$.

This form of embrittlement decreases at higher temperatures, but the use of LAMS may be limited by other properties such as possible swelling at around $400-450{ }^{\circ} \mathrm{C}$ and thermal creep, structural instabilities, softening and non-hardening embrittlement at still higher temperatures $[19,20]$. Thus the temperature window for LAMS could be very limited. Another key issue is irradiation creep that operates over a very wide temperature range; and this phenomena will make fusion structures inherently dimensionally unstable even if high levels of helium and hydrogen do not trigger rapid void swelling.

\subsubsection{Development of radiation resistant alloys: NFA}

Fortunately it may be possible to mitigate or avoid many of the problems described above by designing new alloys to manage both helium and displacement damage, while at the same time optimizing properties such as creep strength and rupture life. The concept is simple and is being explored in the nanostructured ferritic alloys. The NFA typically have higher non-transformable $\mathrm{Cr}$ levels of 12 to $14 \%$ (or more) lying beyond the $\gamma$-loop along with smaller quantities of $\mathrm{W}(2 \%)$ and $\mathrm{Ti}$ $(0.5 \%)[6,17]$. The last is a critical element [21]. NFA are processed by mechanically alloying powders by ball milling, and hot powder consolidation by extrusion or hot isostatic pressing. They are characterized by of nm-scale $\mathrm{Y}$-Ti-O enriched coherent transition phase precipitates and larger incoherent mixed oxide particles. The NFA precipitate and dislocation and grain structures depend on consolidation temperatures and post-consolidation thermal-mechanical heat treatments. For final processing at about $1100^{\circ} \mathrm{C}$ NFA contain more than $10^{23} / \mathrm{m}^{3} 2-3 \mathrm{~nm}$ coherent precipitates in $1-10 \mu \mathrm{m}$ grains with high dislocation densities [21-23]. Extruded alloys are highly textured, with elongated grains having typical aspect ratios of 10/1.

Helium is most damaging if it collects at grain boundaries, or in sufficiently large matrix bubbles that nucleate growing voids. In particular, grain boundary helium produces brittle IG fracture at lower temperatures and nucleation sites for the stress driven growth of cavities that lead to rapid creep rupture at low strains at high temperatures [24]. In principle, a high density of nm-scale precipitates such as the Y-Ti-O enriched precipitates in NFA can serve as trapping sites for helium. If the helium can be maintained in the form very fine scale cluster-nucleated bubbles, the grain boundaries will be protected and void swelling avoided. In addition to helium management, the precipitates may also act as sinks or traps for point defects, reducing the accumulation of radiation displacement damage; and, perhaps, even lowering irradiation creep rates.

Further, the nm-scale precipitates greatly enhance the thermal creep strength and rupture lifetimes of alloys, elevating the potential service window for iron-based alloys to $700^{\circ} \mathrm{C}$ or above. This is generally outside the range of concern for displacement induced radiation damage. However, hydrogen embrittlement may occur and the elevated chromium levels that still provide good protection against oxidation and corrosion can lead to the formation of embrittling phases like $\alpha^{\prime}$ and $\sigma$. There is also evidence that by eliminating coarser scale inclusion and carbides, that act as trigger-particles for locally brittle cleavage fracture, good fracture toughness can be maintained up to very high levels of 
alloy strength. Finally, the fine scale microstructures also appear to be resistant to both flow localization and cyclic strain softening, perhaps, by enhancing the cross-slip of dislocations

The discussion above emphasized the role of the nm-scale precipitates. However, the balance of other microstructures features in NFA is also critical. Depending on the processing details, NFA can have very fine $\mathrm{nm}$ to $\mu \mathrm{m}$ scale re-crystallized grains along with a high dislocation densities introduced during hot consolidation and/or post consolidation deformation. The nm-scale precipitates, dislocations and dislocation substructures all interact and evolve during processing (and likely service) in tandem. For example, the precipitates can pin dislocations and grain boundaries, which in turn provide fast diffusion paths for particle coarsening. Dislocation structures are also critical in trapping helium in bubbles as well as potentially transporting it to grain boundaries.

The microstructures of NFA are controlled by complex non-equilibrium phenomena occurring during both processing and service. Processing involves first ball milling $\mathrm{Cr}, \mathrm{W}$, Ti iron alloy and $\mathrm{Y}_{2} \mathrm{O}_{3}$ powders. It has been recently shown that the $\mathrm{Y}$ and $\mathrm{O}$ dissolve in the $\mathrm{Fe}-\mathrm{Cr}$ matrix during mechanically alloying and re-precipitates as coherent clusters during subsequent hot consolidation of the powders by extrusion or hot isostatic pressing. The nanostructures depend on the combination of the alloy composition and details of all the processing variables, ranging from the milling energy and time, to the consolidation method and time-temperature history, to any post-consolidation thermal-mechanical treatments. How best to manipulate the nanostructures is not understood, nor is the optimal arrangements of the features for irradiation service. The $\mathrm{nm}$-scale non-equilibrium coherent transition phases are metastable and will evolve in service at rates controlled by combinations of non-equilibrium thermodynamics and complex kinetics. Recent studies suggest that the features are expected to be thermally stable for long periods of time at temperatures up to $800^{\circ} \mathrm{C}$ or more, at least in the absence of stress. However, additional complications arise under irradiation due to both ballistic local resolutioning of solutes and coupled inverse Kirkendall type solute-defect fluxes as well as radiation enhanced diffusion. These mechanisms may act in opposition or a parallel-coupled fashion, and the overall significance of the irradiation driven character of the evolutions is not understood. Outcomes can range from accelerated coarsening and phase instabilities to further refinement of the larger oxide features. However, high dose data from fission reactor irradiations suggests that the nanostructures are generally stable, and are not rapidly driven to a degraded coarser-scale state.

It is not yet known how effective the nanoscale clusters will be at managing either helium or displacement damage. Based on experience in other alloy systems, if the 'wrong' type of precipitates trap helium, the corresponding bubbles can act as unwanted nuclei for growing voids. Recently a multiscale modeling effort has been initiated to assess the factors that will control the amount and distribution of helium in stable fine scale bubbles versus collection grain boundaries. Atomistic molecular dynamics and Monte Carlo tools are being used to map key energy interaction parameters, reaction mechanisms and paths. This information is then incorporated in kinetic diffusion, transport and fate cluster dynamics models to assess helium partitioning to various microstructural sites. Preliminary results, using bcc copper with a positive misfit strain as a surrogate for the NFA nm-scale precipitates, show that the interface and elastic properties of the clusters play a key, and non-intuitive, role in helium and defect trapping. Further, dislocations can also play an important role in forming a population of very small bubbles. These results suggest that helium binding energies to cluster and dislocation sites on the order of $0.6 \mathrm{eV}$ are sufficient effectively protect grain boundaries at $600^{\circ} \mathrm{C}$. However, current models must rely on approximate embedded atom $\mathrm{Fe}$ and $\mathrm{Fe}-\mathrm{Cu}$ alloy potentials that do not even exist for the $\mathrm{Y}-\mathrm{Ti}-\mathrm{O} / / \mathrm{FeCr}$ system of interest to NFA. Thus efforts are underway to use $a b$ initio methods to develop alloy parameters for the $\mathrm{Y}-\mathrm{Ti}-\mathrm{O} / / \mathrm{FeCr}$ system and use these results to develop alloy potentials tailored to the problem being addressed.

\subsubsection{Modeling approach for addressing challenges to material performance}

Issues related to modeling complex engineering properties that specifically pertinent the leading candidate LAMS and NFA are briefly outlined below. A hierarchical framework for modeling static 
deformation and fracture properties, outside the creep and fatigue regime, has been developed for low alloy reactor pressure vessel steels, and can be applied to LAMS and NFA. Modeling efforts must be linked to a wide variety of experiments to understand mechanisms, measure key parameters, and validate and calibrate model predictions.

- Modeling the relation of key irradiation and material variables to the microstructural evolution of hardening features and the coarser structures controlling local fracture properties. In LAMS the pertinent hardening features are small defect clusters (primarily, loops and cavities) and precipitates (primarily, $\alpha$ ' and possibly fine scale carbides) of reasonably well-known character. The local fracture properties are controlled by coarser scale features, like grain boundary carbides, that are generally stable at lower temperatures. However, they are unstable at the higher end of the proposed temperature window for LAMS. It is hoped that NFA will be stable under irradiation, and modeling will focus on the nano-microstructural stability

- The relation between microstructure local micro-constitutive properties. Integrated multiscale models combine sub-models of dislocation-obstacle interactions, dislocation based strength superposition simulations and evolutionary internal state variable structural evolution true stress-strain constitutive and plasticity models that can be used in finite element codes. Thus the changes in microstructure are related to changes in the constitutive properties. At higher temperatures, larger scale structural instabilities and softening must also be modeled. Dislocation dynamics models will also provide important insight into phenomena like flow localization, including controlling mechanisms, triggering criteria and spatial length scales. Over the long-term dislocation dynamics may also provide a basis for direct simulations of deformation in complex alloy microstructures.

- Advanced micromechanics models of the relation between the microstructure and local fracture properties, typically represented as microstructurally dependent critically stressed or strained local volume, or the statistical model equivalent. Micromechanical also requires multiscale integration of submodels, such as for the trigger particle nucleated arrest toughness of nano-blunting microcracks in bcc ferritic lattices using dislocation dynamics methods. Again changes in microstructure are related to changes in the local fracture properties. The major pertinent microstructural changes are growth and precipitation of coarser scale brittle grain boundary phases, the effects of helium and hydrogen on the cohesive strength of grain boundaries and the critical stressed volume for intergranular (IG) fracture. The possible role of flow localization in dictating local fracture properties must also be investigated.

- Modeling the relation between the combination of local constitutive and fracture properties and more complex engineering properties like tensile deformation stress strain curves and fracture toughness. These property models link applied stresses and strains to the micromechanical constitutive and fracture models described above though finite element simulations of the development of internal stress and strain field distributions. These fields drive local deformation and fracture processes. Finite element modeling includes stress and strain fields a blunting crack tips that drive the local fracture process and deformation and internal flow patterns that develop at various length scales in tensile tests. These models provide the critical link between the atomic to local microscale processes and continuum level loading conditions that explicitly account for size scales, geometry, loading states and stochastic effects in inherently heterogeneous materials.

- Integration of the modeling elements listed above to provide quantitative and predictive models of the mechanical properties describing deformation and fracture that are fully 
calibrated and verified. These models must include assessment of uncertainties and statistical effects to provide specified confidence intervals as well as mean values.

- Developing advanced structural integrity assessment methods and models to predict the applied stress-strain failure limits of loaded structure based on the property predictions provided by the integrated models described above. Ultimately this should be a seamless part of an advanced effort to model the structural and functional performance of fusion structures and integrated chamber and divertor systems.

The modeling efforts just discussed primarily pertain to what are called static mechanical properties. A critical parallel effort must be initiated on addressing high temperature time-dependent properties, including thermal and irradiation creep, creep rupture, and ductility in the presence of high levels of helium. This topic has not been addressed substantially in this report; and, in large part, it involve a different set of issues, i.e. high temperature helium accumulation on grain boundaries and creep constrained cavity growth. However, the use of advanced computational tools in a comprehensive

program that is linked to experiments should enable a similar general framework to be established for modeling these critical properties as well.

\section{References}

1. E. E. Bloom, et. al., A Roadmap for the Advanced Structural Materials Program, Whitepaper prepared for the US Department of Energy, Oak Ridge National Laboratory (1999)

2. R. E. Stoller, G. R. Odette and H. L. Heinisch, An Integrated Program of Theoretical, Experimental and Database Research for the Development of Advanced Fusion Materials, Whitepaper prepared for the US Department of Energy, Oak Ridge National Laboratory (1999)

3. G. R. Odette, B. D. Wirth, D. J. Bacon and N. M. Ghoniem, MRS Bulletin March (2001) 176

4. S. Jumel, et. al., J. Test. and Eval. 30 (2002) 37.

5. M. K. Miller, D. T. Hoelzer, S. S. Babu, E. A. Kenik, and K. F. Russell, in High Temperature Alloys: Processing for Properties, G. E. Fuchs and J. B. Wahl, Eds., to be published in Met. Trans., 2004.

6. S. Ukai, M. Fujiwara, J. Nucl. Mater. 307-311 (2002) 749.

7. R. E. Stoller, P. J. Maziasz, A. F. Rowcliffe and M. P. Tanaka, J. Nucl. Mater. 155-157 (1988) $1328-1334$.

8. E. A. Kenik and E. H. Lee, in Phase Stability Under Irradiation, J. R. Holland, L. K. Mansur, and D. I. Potter, Eds., AIME, New York, 1981, pp. 493-503.

9. J. D. Hunn, E. H. Lee, T. S. Byun, and L. K. Mansur, J. Nucl. Mater. 282 (2000) 131-136.

10. J. D. Hunn, M. B. Lewis, and E. H. Lee, in Proceedings of the Second International Topical Meeting on Nuclear Applications of Accelerator Technology, American Nuclear Society, La Grange Park, 1998, pp. 375-381.

11. For a review: G. Martin and P. Bellon, Solid State Physics 50 (1996) 189.

12. V. G. Vaks, Physics Reports 391 (2004) 157.

13. N. M. Ghoniem, B. N. Singh, L. Z. Sun, and T. Diaz de la Rubia, J. Nucl. Mater. 276 (2000) 166177.

14. N. M. Ghoniem, S.H. Tong, B. N. Singh, and L. Z. Sun, Phil. Mag. A 81 (2001) 2743-2764.

15. Yu. N. Osetsky, D. J. Bacon, and V. Mohles, Phil. Mag 83 (2003) 3623 - 3641.

16. R. L. Klueh, D. S. Gelles, S. Jitsukawa, A. Kimura, G.R. Odette, B. van der Schaaf, and M. Victoria, J. Nucl. Mater. 307-311 (2002) 455.

17. G. R. Odette, T. Yamamoto, H. J. Rathbun, M. Y. He, M. L. Hribernik, and J. W. Rensman, J. Nucl. Mater. 323 (2003) 313.

18. T. Yamamoto, G. R. Odette, H. Kishimoto, and J.W. Rensman, Fusion Reactor Materials Semiannual Report 7/1 to 12/31/2003, DOE/ER-313/35 (2004) 100-114.

19. R. L. Klueh, P. J. Maziasz, I. S. Kim, L. Heatherly, D. T. Hoelzer, N. Hashimoto, E. A. Kenik, and K. Miyahara, J. Nucl. Mater. 307-311 (2002) 773. 
20. M. J. Alinger, G. R. Odette, and G.E. Lucas, J. Nucl. Mater. 307-311 (2002) 484.

21. M.K. Miller, E.A. Kenik, K.F. Russell, L. Heatherly, D.T. Hoelzer, and P.J. Maziasz, Mat. Sci. Eng. A - Struct. 353 (2003) 140.

22. M.J. Alinger, G.R. Odette, D.T. Hoelzer, J. Nucl. Mater. (2004) in press.

23. H. Kishimoto, M. J. Alinger, G.R. Odette, T. Yamamoto, J. Nucl. Mater. (2004) in press.

24. H. Trinkaus, B.N. Singh, J. Nucl. Mater. 323 (2003) 229. 


\section{Session IV: Expected Advances and Contributions of Computational Science}

\subsection{Objectives of Session IV}

This session focused on the current state of the art in computational science with the goal of providing a perspective on the advances in hardware, software, and algorithms that are expected during the next 5 to 10 years. It included relevant discussion of, and lessons learned from, recent experience with large-scale simulation programs such as the Accelerated Strategic Computing Initiative (ASCI), and the Scientific Discovery through Advanced Computing (SciDAC) initiative which has involved a broad range of computational and physical scientists.

\subsection{Introduction}

The steep and sustained rate of decrease in cost per unit of computing power coupled with the steady growth of scientific simulation infrastructure-fueled from numerous disciplines including materials science - insures that scientific simulation and data science will play increasingly important roles in the design and evaluation of materials for advanced fission and fusion reactors. Simulations of increasing economy, capability, and reliability will eventually "cross the line" of what experimental science can deliver in a given domain, and it is timely to attempt to assess how far out is that crossing in several critical areas of materials design.

Simulation and data science can be expected to reduce the number of physical experiments required to support the development of materials for advanced reactors, but by no means to eliminate all expensive experiments. They may also be expected to reduce the latency of an experimental program leading to reactor design and qualification and to reduce the range of experiments that need to be conducted and the cost of performing individual experiments. However, no combination of information technologies foreseeable in the coming decade of interest is expected to completely bypass major experimental investment, for reasons that are described in this section. In fact, additional experiments, outside of those that would be required in a reactor development path that largely ignored simulation, may be recommended in order to develop validated simulation tools and databases.

If all of the phenomena important to a predictive science of materials were reliably understood today at the appropriate scales from a theoretical point of view, then a "worst case" roadmap for simulation of materials could be laid out with some confidence, based on the known computational complexities of contemporary algorithms for attacking each problem, and extrapolations of computer processing power. However, the multiscale nature of materials science presents us at the time of writing with a veritable patchwork of simulation capabilities for different regimes, and significant uncertainty in how best to tie them together. Thus, extensive interplay between theory, experiment, and simulation will be required to make advances worthy either of the best traditions of science and engineering or of capital investment in advanced reactors in the billion-dollar range.

Accompanying this cautionary conclusion are several reasons for optimism, emerging from a workshop that united materials scientists with mathematical and computer scientists. First, a program for materials design for advanced reactor concepts, while arguably presenting unique physical challenges, has much in common with materials programs for military and other industrial technologies, and multi-pronged research efforts exist in these sectors that provide both patterns and applicable tools. Second, the Department of Energy has had since 1996 a simulation program leading to the certifiabilitiy of nuclear weapons [1]. Lessons of enormous importance in terms of hardware platforms, software engineering, validation and verification, and the sociology of sizeable multidisciplinary code teams, have been learned from this effort, many of which are transferable, with a much compressed learning curve, to a simulation program for advanced materials [1]. Third, the "science of simulation" is itself rapidly improving. Its rising tide of capability lifts all boats. While materials science has unique modeling challenges, computational materials science shares kernels (such as fast Poisson solvers and 
dense eigensystems) and information infrastructure (such as distributed data bases and visualization environments) with numerous other applications. As investments are made continually in simulation and information technology, they are leveraged by materials scientists. Finally, the probability is high that stimuli from challenges in materials science will spur developments in numerical algorithms, scientific data mining, or some other aspects of the information science infrastructure that will lead to punctuated advances in the predictive capability of computational materials science. Such "breakthroughs" are by definition not themselves predictable, but they are in historical evidence in situations where applied mathematicians and computer scientists are driven by new and concrete challenges, of which the modeling of materials provides many.

In short, a balanced investment in simulation and experimental approaches is recommended, in which a premium is accorded to potentially high-payoff issues at the traditional location of the interface between materials scientists and engineers with applied mathematicians and computer scientists, while not neglecting more generic simulation infrastructure. Commissioned in isolation, modelers are likely to constrain the equations they consider, the types of interactions they include, the types of boundary conditions they impose, the amount of data they can access, etc., because of their understanding of currently available technology. To make progress on a timescale that allows simulation to inform and interact with experiment in a lively way, it is necessary to present the multiscale, data-intensive challenges of materials science to computational scientists, so that best practices evolve. To keep knowledge flowing in the other direction, it is necessary for modelers to continually seek to apply and adapt new methodologies that optimize the representation and solution of problems, the comprehension of data, and the integration of techniques developed independently into larger computational systems.

The remainder of this section outlines some of the principal tools that applied mathematics and computer science bring to computational materials science, noting some special challenges, following [2]. Following [1], it then briefly discusses validation and verification in materials science and software engineering for complex materials simulation. Some specific related contemporary efforts to which to turn for examples in shortening the development curve, following [3] and [4] are mentioned next. We conclude by noting some current high watermarks in first-principles materials simulation that were barely conceivable until they were recently accomplished. These provide both exemplars and encouragement for a major simulation initiative in advanced materials design.

\subsection{Mathematical Tools for Computational Materials Science}

Mathematics is the bridge between physical systems and computer simulations of those systems. The starting point for a computer simulation in materials science is a mathematical model such as quantum or classical molecular dynamics, dislocation dynamics, kinetic equations for growth or decay, or continuum mechanics, to name a few. For such a model, there are three categories of mathematical tools to bring to bear in order reliably to use computer simulations.

(1) Model analysis A mathematical model should be internally consistent and well posed for its computer representation to be well defined. Do the equations have a unique solution over the range of physically meaningful inputs? Do small changes in the inputs lead only to small changes in the solution, or, as an indication of likely trouble, could a small uncertainty be magnified by the model into large variability in the outcome? Do quantities represent actual states of the system or are they to be interpreted in a statistical sense only?

(2) Approximation and discretization For many systems, the number of unknowns in the mathematical model that is rigorously set up is larger than can be represented or manipulated computationally, and perhaps even infinite, as when the solution is a field defined over the continuum variables of space, time, and phase. In such cases, it is necessary to approximate the high dimension of the system with a possibly large but finite and manipulable number of unknowns. The mathematical issues for this 
discretization process include: (1) the convergence of the discrete approximation to the original equations as the number of computational unknowns increases in regimes where convergence is expected and (2) the behavior of the approximation with respect to singularities or other features of the underlying system perhaps not easily represented.

(3) Solvers and software Once the physical problem has been reduced to the solution to a finite number of equations for a finite number of unknowns, how does one make best use of the computational resources to calculate the solution to those equations? Issues at this stage include the development of optimally efficient algorithms, and the mapping of computations onto a complex hierarchy of processors and memory systems.

Although these three facets represent distinct mathematical specialties, they arise, explicitly or implicitly, in building any simulation. The choice of appropriate mathematical tools can make or break a simulation code, in terms of validity, flexibility, and affordability. For example, over the five decades the era of computational simulation, a change in algorithms for evaluating the electrostatic potential induced by a charge distribution, a typical MD kernel, has been reduced in cost from a polynomial to a linear function in the number of discrete charges or points of evaluation. The improvement resulting from this algorithmic speedup exceeds by an arbitrary factor that resulting from the hardware speedup due to Moore's Law over the same length of time [2]. The improvement due to Moore's Law over any fixed period is a constant, whereas the savings in arithmetic and data movement costs from superior algorithms, such as multigrid [5] or multipole [6] is a function or the problem size - the bigger the problem, the greater the savings. The algorithmic improvements leading this factor are all based on a fundamental mathematical property of the underlying model - namely, that the function describing electrostatic coupling between disjoint regions in space is very smooth. Expressed in the right way, this coupling can therefore be resolved accurately with little computational effort. The various improvements in algorithms for computing electrostatic potential are related to both discretization methods and solution algorithms that exploit the known smoothness.

This is an example of the general principle that pervades mathematical science that the more foreknowledge of the properties of the problem and solution you can build into a solution algorithm, the faster and more accurate it is. Examples include the use of "importance sampling" in Monte Carlo [7], reducing the dimensionality of a problem due to geometrical symmetry and building known symmetries into the set of basis functions.

As expectations for computational materials science progress from simple "insight" into actual numbers to guide policy and investment, there is an increase in the complexity of virtually all aspects of the modeling process. The scientists and engineers contributing to this report identified several areas of applied mathematics for which research and development is needed must accompany the rapidly increasing expectations on state-of-the-art computational science. They fall into the following three categories.

\subsubsection{Managing model complexity}

Scientists want to use increasing computing capability to improve the fidelity of their models. For many problems, this means introducing models with more physical effects, more equations, and more unknowns. When developing comprehensive models, the goal is to develop a combination of analytical and numerical techniques to better represent problems dependent on multiple physical processes or mechanisms. These techniques may range from analytical methods to determine how to break a problem up into weakly interacting components, to new numerical methods for exploiting such a decomposition of the problem to obtain efficient and accurate discretization in time. A similar set of issues arises from the fact that many systems of interest have processes that operate on length and time scales that vary over many orders of magnitude. Multiscale modeling addresses the representation and interaction of behaviors on multiple scales so that results of interest are recovered without the 
(unaffordable) expense of representing all behaviors at uniformly fine scales. Approaches include the development of adaptive methods, i.e., discretization methods that can represent directly many orders of magnitude in length scales that might appear in a single mathematical model, and hybrid methods for coupling radically different models (continuum vs. discrete, or stochastic vs. deterministic), each of which represents the behavior on a different scale. Uncertainty quantification addresses issues connected with mathematical models that involve fits to experimental data, or that are derived from heuristics that may not be directly connected to physical principles. Uncertainty quantification uses techniques from fields such as statistics and optimization to determine the sensitivity of models to inputs with errors and to design models to minimize the effect of those errors.

\subsubsection{Discretization of spatial models}

Many simulations of radiation damage in materials have the equations of structural dynamics or radiation transport, or both, as core components of their mathematical models. Computational structural dynamics and transport and kinetic methods have as their goal the development of the next generation of spatial discretization methods for these problems. Issues include the development of discretization methods that are well suited for use in applications that involve simulating multiple physical mechanisms without loss of accuracy or robustness. Historically, the use of low order discretization of partial differential equations predominates. Such discretizations lead to very large and sparse systems of algebraic equations, which overwhelm the storage and bandwidth capabilities of likely future computational platforms, relative to floating point processing. High-order discretization and alternative formulations such as integral equations should be considered to address trends in computer architecture. Meshing methods specifically address the process of discretization of the computational domain, itself, into a union of simple elements (cf. [8]). This process is usually a prerequisite for discretizing the equations defined over the domain. This area of work includes the management of complex geometrical objects arising in technologically realistic devices. Historically, many insights into the strength and damage resistance of materials have come from geometrically simple methods with periodic boundary conditions.

\subsubsection{Managing computational complexity}

Once the mathematical model has been converted into a system of equations for a finite collection of unknowns, it is necessary to solve the equations. The goal of efforts in the area of solvers and "fast" algorithms is to develop algorithms for solving these systems of equations that balance computational efficiency on hierarchical multiprocessor systems, scalability (the ability to use effectively additional computational resources to solve increasingly larger problems), and robustness (insensitivity of the computational cost to details of the inputs). An algorithm is said to be "fast" if its cost grows, roughly, only proportionally to the size of the problem. This is an ideal algorithmic property that is being obtained for more and more types of equations. Discrete mathematics and algorithms make up a complementary set of tools for managing the computational complexity of the interactions of discrete objects. Such issues arise, for example, in traversing data structures for calculations on unstructured grids, in optimizing resource allocation on multiprocessor architectures, or in scientific problems in areas such as bioinformatics that are posed directly as "combinatorial" problems.

As of the time of writing, standard tools of reliable quality, unlikely to be significantly improved upon, exist for dense and some sparse linear systems (e.g. [9,10]), moderate scale systems of ODEs (to allow "method-of-lines" solution of time-dependent PDEs as well as discrete systems, e.g. [11]), small-scale optimization (e.g. [12]), and small-scale eigenanalysis (e.g. [13]). Numerical analysts are steadily extending the domain of fast algorithms with reliable, tunable accuracy to special systems governed by PDEs, including potential problems, wave Helmholtz problems, Maxwell's equations, the heat equation, Navier-Stokes in laminar or narrowly defined turbulent regimes, and linear elasticity. Such PDEs have numerous kernel applications in materials science; for instance, fast algorithms for potential problems are at the heart of dislocation dynamics and molecular dynamics. In problems where they do not directly apply (due to problem complexity), they may be used to construct preconditioners. 
Materials design is ultimately in the quantum regime and involves inverse problems, not simply "forward" PDEs. Fast algorithms play an important supporting role, but breakthroughs in simulation complexity is likely to come only from improved physical modeling.

The goals of scientific software in these areas should be to allow problem specification at multiple levels of abstraction, to state working ranges of accuracy and complexity, and to promote correctness testing and performance profiling.

\subsection{Computer Science Tools for Large-scale Materials Simulation}

The role of computer science in large-scale simulation of materials is to provide tools that address issues of algorithmic complexity, computational performance, navigation of the results of simulation, integration of the data of simulation and experimental observation, and human comprehension of that data. These issues cut across the computer science disciplines. An integrated approach is required to solve the problems faced by applications.

One of the major challenges for applications is the code complexity of turning a mathematical model into an effective simulation. There are numerous reasons for this. Often, even after the principles of a model and simulation algorithm are well understood, too much effort still is required to turn this understanding into practice because of the low level at which code is designed. Current programming models and frameworks do not provide sufficient support to allow domain experts to be shielded from details of data structures and computer architecture. Even after an application code produces correct scientific results, too much effort still is required to obtain high performance. Code tuning efforts needed to match algorithms to current computer architectures require subtle analysis and experimentation.

Once an application runs effectively, often the next hurdle is saving, accessing, and sharing data. Once the data are stored, since ultrascale simulations often produce ultrascale-sized datasets, it is still too difficult to process, investigate, and visualize the data in order to accomplish the purpose of the simulation - to advance the science. These difficulties are compounded by the problems faced in sharing resources, both human and computer hardware.

Despite these hurdles, prospects for placing usable computing environments into the hands of materials and radiation experts are improving. In the last few years, there has been a growing understanding of the problems of managing complexity in computer science, and therefore of their potential solutions. For example, there is a deeper understanding of how to make programming languages expressive and easy to use without sacrificing high performance on the sophisticated, adaptive algorithms.

Another relevant trend is the success of component-oriented software; such "components" have allow simulation experts to focus their own expertise on their science while exploiting the newest algorithmic developments. Many groups in high-performance computing have tackled these issues with significant leadership from the Department of Energy, most recently through the SciDAC program. Fully integrated efforts are required to produce a qualitative change in the way application groups cope with the complexity of designing, building, and using ultrascale simulation codes.

One of the drivers of software complexity is the premium on performance. The most obvious aspect of the performance problem is the performance of the computer hardware. Although there have been astounding gains in arithmetic processing rates over the last five decades, users often receive only a small fraction of the theoretical peak of processing performance. There is a perception that this fraction is, in fact, declining. This perception is correct in some respects. For many applications, the reason for a declining percentage of peak performance is the relative imbalance in the performance of the subsystems of high-end computers. While the raw performance of commodity processors has followed Moore's Law and doubled approximately every 18 months, the performance of other critical parts of the system, such as memory and interconnect, have improved much less rapidly, leading to less- 
balanced overall systems. Solving this problem requires attention to system-scale architectural issues. Different kernels are optimized with different balances of hardware resources, such as the ratio of bytes per second delivered by the memory system to flops per second performable by the processor, or the product of the latency and bandwidth of message passing, which determines the break even packet granularity. A general-purpose ultrascale computer must support diverse kernels. Simple number metrics do not characterize overall computer performance on a complex application anymore than speedometer peak characterize average speed in dense traffic. The discrepancy between the latter two speeds does not mean that the car does not need a powerful engine on some occasions.

As with code complexity issues, there are multiple on-going efforts to address hardware architecture issues. A single architectural convergence point, such as that occupied by current commodity-based terascale systems, may not be the most cost-effective solution for all users. A comprehensive simulation program requires that several candidate architectural approaches receive sustained support to explore their promise.

Performance is a cross-cutting issue, and computer science offers automated approaches to developing codes in ways that allow computational materials and radiation scientists to concentrate on the physics. For example, techniques that allow a programmer automatically to generate code for an application that is tuned to a specific computer architecture address both the issues of managing the complexity of highly tuned code and the problem of providing effective portability between highperformance computing platforms. Such techniques begin with separate analyses of the "signature" of the application (e.g., the patterns of local memory accesses and inter-processor communication) and the parameters of the hardware (e.g., cache sizes, latencies, bandwidths). There is usually plenty of algorithmic freedom in scheduling and ordering operations and exchanges while preserving correctness. This freedom should not be arbitrarily limited by particular expression in a low-level language, but rather chosen close to runtime to best match a given application-architecture pair. Similarly, the performance of $\mathrm{I} / \mathrm{O}$ and dataset operations can be improved significantly through the use of well designed and adaptive algorithms.

The scope of computer science includes understanding the results of a computation. Ultrascale datasets are too large to be grasped directly, that is, as tables of numbers. Applications currently rely on a variety of tools to attempt to extract patterns and features from the data. Computer science offers techniques in data management and understanding that can be used to explore data sets, searching for particular patterns. Visualization techniques help scientists explore their data, taking advantage of the unique human visual cortex and visually stimulated human insight. Current efforts in this area are often limited by the lack of resources, in terms of both staffing and hardware.

Understanding requires harnessing the skills of many scientists. Collaboration technologies help scientists at different institutions work together. Grid technologies that simplify data sharing and provide access to both experimental and ultrascale computing facilities allow computational scientists to work together to solve the most difficult problems facing the nation today. Although these technologies have been demonstrated, much work remains to make them a part of the typical scientist's toolbox. Key challenges are in scheduling multiple resources and in data security.

Critical computer science issues must be addressed in parallel with physical and mathematical issues to leverage progress in the latter. Visual data exploration and analysis considers understanding the results of a simulation through visualization. Computer architecture looks at the systems on which ultrascale simulations run and for which programming environments and algorithms provide software tools. Programming models and component technology develop techniques for turning algorithms into efficient, maintainable programs. Resource sharing makes data and processing resources available to the entire research community and promotes collaboration. Software engineering and management is about disciplines and tools - many inherited from the industrial world of software engineering (cf. [14-16])to manage the complexity of code development. Data management and analysis addresses the challenges of managing and understanding the increasingly staggering volumes of data produced by 
ultrascale simulations. Performance engineering is the art of achieving high performance, which has the potential of becoming a science. System software considers the basic tools that support all other software. The simulation of materials need not take the lead in research any of these topics; they are generic and are being aggressively pursued in programs that leverage all computational disciplines. Materials scientists need to remain aware of relevant progress and open to evolving their processes to better take advantage of new technologies and workforce skills in these areas.

The emergence of ultrascale computers costing in the hundreds of millions of dollars invites comparisons between computational facilities and high-end experimental facilities, such as the light sources of experimental physics. Just as a beamline requires specialized experimental end stations to allow the physicist to efficiently employ the resource without an intimate knowledge of the inner workings of the entire accelerator, "end station" computational environments need ultimately to be developed for production use of simulation facilities by materials scientists. The goal of packaging verified and validated problem-solving environments for production use does not contradict the interdisciplinary approach advocated above, in which materials scientists and computational scientists must first labor together at the interfaces of their disciplines to develop such computational tools.

While materials scientists may not be the principal drivers in any of the mathematical or computational research campaigns from which they stand to benefit, issues specific to materials science could be among the most useful drivers in some of the relevant technologies. The ultimate vertical interrelatedness of all these issues in a simulation environment points to the potential synergy of integrated efforts in scientific discovery in materials science and in the science of simulation itself.

\subsection{Verification and Validation for Materials Models}

A computational simulation of some natural phenomenon involves an intermediary mathematical model. The credibility of the calculation depends on verification that the model is correctly solved, and validation that the model accurately captures the natural phenomenon over an intended physical regime $[17,18]$. Without both, there is no reason to believe the calculation. Five verification techniques are commonly used: comparison with analytic test problems, convergence rate studies, use of "manufactured solutions" [19], monitoring of "conserved quantities" and expected behavior, and comparison with comparable codes. While these are all useful and mostly complementary, each has limited effectiveness, and even their union is not sufficient to verify completely that the code is solving the model accurately. While potentially rigorous for unit tests, most are not very useful for the verification of large, complex codes. The development of better verification methodologies is crucial for improving the quality of computational predictions in materials science and in other realms.

The reliability of a calculation also depends on how accurately the mathematical model in the code captures the main features of the phenomenon being studied. The only way this can be established is by comparison with observations and experiments of the natural world. There are at least three types of validation experiments: passive observations, controlled experiments mostly carried out for other purposes such as scientific discovery, and controlled experiments carried out mainly for validation. Geophysical and astrophysical codes rely predominantly on passive observations, and validation poses special challenges. Most validation is now done using existing experimental data gathered a part of a scientific investigation for scientific discovery. Such data are often not well suited for validation. The data generally do not exercise enough features or gather enough data to test many of the key elements of the code, and often lack adequate resolution. Also, codes can frequently be made to fit a nearly arbitrary data set regardless of the problems or consistency of the data.

The best validation tests are accomplished using experiments explicitly designed for validation. Such experiments generally provide data that is more complete and more appropriate for validation. In addition experimental facilities designed primarily for validation can often be smaller and cheaper with shorter setup times and faster turnaround times than conventional experimental facilities. A validation 
experiment does not have to test a specific system or component, but merely to exercise and test major portions of the code.

Codes that can successfully predict experimental results before the experiments are performed provide the most reliable predictions. A paradigm shift is needed in the experimental community before validation experiments become common. Both experimentalists and their sponsors now fail to understand the importance and value of experiments designed primarily for validation. Obtaining funding for a validation experiment is very challenging.

The errors for a complicated materials model are a nonlinear convolution of the constituent components and their integration. The materials community has a good record with regard to validation. However, verification and validation of codes with many different components and models is more demanding than for a simpler code that only treats a few effects. Each component should be verified and validated independently, and then the integrated system has to be verified and validated.

Validation facilities are required that are able to test all relevant portions of the simulations for advanced materials. For fission materials, one will need to have facilities with prototypical neutron spectra, fluxes, and fluences. Simulation will likely allow one to use far fewer experimental tests, but tests of all relevant models for the important parameter regime will be essential. This includes high temperature material conditions with sufficient exposure time that long-term effects can be identified and characterized. For fusion materials, again facilities with prototypical neutron fluxes, spectra, and fluences will be needed. Simulation will likely allow many fewer materials tests than a completely empirical approach, but the models in the codes will need validation.

There was insufficient information presented at the workshop to allow specific comment on the degree to which computer simulation can reduce the requirements for facilities compared to a completely empirical approach, but it is clear that a substantial reduction could potentially be achieved. However, as noted above, validation is essential, and special experimental facilities are needed for validation.

\subsection{Software Engineering for Complex Materials Simulations}

The DOE/NNSA Accelerated Strategic Computing Initiative (ASCI) program offers a perspective on the scale of effort needed to develop an integrated simulation capability for material radiation damage due to neutrons. The goal of the ASCI program is to produce a family of codes that can predict the performance of a nuclear weapon and provide confidence in the national nuclear stockpile without full-scale tests. The program was started in 1996, underwent an intensive review by the JASONS in 2003, and continues today. The total cost of the program has been about $\$ 700 \mathrm{M}$ a year, including all of the software and hardware costs. The development of a typical massively parallel application code that includes all of the relevant physics involves a team of about 25 people at cost of about $\$ 8 \mathrm{M} /$ year and takes between 8 and 10 years to bring a state in which it can be turned over for production use. This is consistent with the experience of the general Information Technology industry [1]. Validation has been a key activity and validation experiments are funded separately. Validation is an ongoing process. Validation data has been obtained from archives of past nuclear tests and ongoing experiments on above-ground experiments including tests with high explosive facilities like the DAHRT facility at LANL and inertial confinement facilities like the OMEGA laser facility at the University of Rochester and, in the future, the \$3B National Ignition Facility (NIF) being constructed at LLNL. The details of a weapons code are classified but involve many different tightly coupled physics effects at multiple space and time scales.

The ASCI program made a number of mistakes on the path to success, and the materials science community can and should learn from the ASCI experience [1, 20]. A major mistake of ASCI was failing to account for the differences in code development paradigms as one scales from small code groups of a few staff developing a code with only a few major effects to a large group (20 or more) developing a multi-effects code. Success requires organizing the effort into a project structure while 
retaining the flexibility to do the necessary R\&D to develop new physics capability. Another mistake of ASCI was to set schedules that were much too optimistic. The first milestone was set at about 3.5 years after its start, while the actual time required to develop the capability to meet the first milestone was 8 years. Only those projects that had a 5-year head start prior to the formal beginning of ASCI succeeded. The successful ASCI projects emphasized the following management principles:

- Build on the successful code development history and prototypes for your organization.

- Make good people in a good team the top priority.

- $\quad$ Run the code project like a project.

- Identify, manage, and mitigate risks.

- Determine the schedule and resources from the requirements.

- Maintain strong customer focus.

- Better physics is much more important than better computer science.

- Use modern - but proven - computer science.

- Invest in team skill development.

- Use "best practices" to improve software quality rather than "rigid processes."

- Validation and verification are essential.

Unsuccessful projects failed to respect one or more of these principles. For example, more than one-half of the ASCI code projects did not succeed in meeting their initial milestones.

Materials simulation for advanced reactors has a need for multiscale modeling of phenomena that involving multiple physical mechanisms that is comparable to that of the stockpile stewardship program. The scale of computation involved, and the requirement for high confidence in the simulation results are also similar. Therefore, the principles for code development listed above undoubtedly will apply.

\subsection{Program Models for Advanced Reactor Materials Design}

From presentations and discussions at the workshop, it is clear that the computational materials science effort at the Department of Energy intends to have a broad impact on the materials selection, development, and design of advanced reactor systems. In developing a comprehensive roadmap for the performance prediction (including failure) of nuclear materials, planners may gain much from prior experiences in related structural materials fields. Notably, the Defense Advanced Research Project Agency (DARPA), together with Air Force, Navy and contractor teams led by Boeing, Pratt and Whitney and General Electric, is engaged in the "Accelerated Insertion of Materials" (AIM) initiative, which has evolved a methodology that may be useful. That initiative seeks to develop and insert into service, aerospace structural metals and composites in time spans that are considerably shorter than the 15 - to 20 -year periods that are common today.

The AIM program was founded to bring about three systemic changes to the material development community: 1) revolutionize the way system designers and materials engineers interact, 2) achieve a leap forward in the application of computational materials science and integration with design engineering tools, and 3) create an environment where the design/materials team can learn from and build on previous developments.

The AIM effort is structured around the idea that development of a "designer knowledge base" that incorporates design ranges permitted for materials, reliability, manufacturing, reproducibility, lifetime management and other essential information is today a time-consuming, largely experimental, and limiting endeavor for structural materials insertion. The effort further recognizes emerging efforts in 
materials modeling that are leading to important improvements in specific areas (e.g., materials process simulation, mechanical behavior prediction, and specific physical event simulation). Having these properly integrated with experiments will lead to developing designer information more rapidly and efficiently. The AIM initiative has already examined the required structure, technical content, and fidelity of the designer knowledge base to drive efficient use of models and experiments for aerospace materials, and has identified required advances in modeling capability for specific classes of predictive challenges. Critical to that effort is understanding how to effectively use materials models, how to link them across various length and time scales, how to couple them with an optimized series of experiments to yield the appropriate information for the designer, and how to manage error and uncertainty across the effort. Validation of the developed approaches has been integral to the developed process. Further, the initiative acknowledges that the both near-term tools in hand, and longer-term technical advances must fit seamlessly into the framework.

The approach has been to build a series of linked simulation tools that, when coupled with selected experiments, provide the necessary information to the designer more rapidly and accurately than the current empirically based procedure. Aspects of the approach were pioneered by the Steel Research Group at Northwestern University and these were used to establish the required microstructural and property simulation models. The approach linked process/structure/property/performance models for selected alloy systems and extended microstructural evolution tools that use existing thermodynamic/diffusion codes coupled with a precipitation model specifically developed for superalloys. Design-critical turbine engine disk properties (burst, creep/rupture, and notch fatigue) were analyzed and modeled by teams selected to combine phenomenological expertise with superalloy experience. The issue of variability in these and other characteristic properties, such as those generated in acceptance testing, was addressed. Based on comparisons of predictive capability with the design database, a new and much smaller test matrix for the certification of a material was defined that increases speed and reduces cost. The integration of the AIM toolset into the design system architecture was accomplished through the iSIGHT platform.

Finally, several pacing areas for further scientific advance were identified through the initiative. Accurate modeling depends upon extraction and storage of microstructural information and its spatial and temporal evolution; thus there is an ongoing materials representation need. Next, the AIM methodology depends upon materials models having sufficient fidelity and robustness to confidently predict processing, microstructure and mechanical properties. These are expected to continuously evolve in the materials community. Lastly, design of a system must account for uncertainty inherent in materials behavior, materials models and manufacturing processes. Thus, the initiative can only move forward with appropriate methods for uncertainty and error management.

A different research domain with similar needs to synthesize multiscale information to support a systems-based research approach is combustion. In this community, an open source multiscale informatics toolkit, the Collaboratory for Multiscale Chemical Science (CMCS) addresses a number of issues core to the emerging concept of knowledge grids, including provenance tracking and lightweight federation of data and application resources into cross-scale information flows. The CMCS portal is currently in use by a number of high-profile pilot groups and is significant in enabling their efforts to improve and extend community maintained chemical reference information.

In materials science, as in combustion science, an understanding of system scale phenomena requires more than simply applying one type of computation, with increased computing power, across scales. Different physical phenomena dominate system dynamics at these different scales, leading to a variety of conceptual models, and associated experiments and computations, relevant in the different regimes. One of the major bottlenecks in contemporary multiscale research is in the passing of information from one level to the next in a consistent, validated, and timely manner. Traditionally, this information flow has been accomplished at the community level through the research literature. Increasingly, the literature is supplemented by community databases. In more project-oriented disciplines, collaboratory and grid models for virtual organizations are starting to play a role. However, 
these approaches do not yet scale well with respect to community, process, and data/metadata heterogeneity.

The CMCS is designed to address many of these challenges and provide rich group-level collaboration capabilities, facile bi-directional data flow between groups and larger communities and between communities, and community-level review and curation mechanisms. The CMCS project was initiated in 2001 with a long-term vision of multiscale science enabled by modern informatics, and a commitment to realizing this vision in support of combustion research.

For data and metadata management, CMCS employs the scientific content management services developed within the DOE-funded Scientific Annotation Middleware (SAM) project, which provides a range of capabilities for storing and retrieving data and metadata, searching, versioning, locking, and providing access control, as well as extensible mechanisms for extracting metadata from files, performing translations, and managing provenance and other data relationships. CMCS's design allows integration of all information related to an experimental case. The repository can store data and associated notes, images, documents, and other material in any format and store all relationships between them. In addition to the tools for managing data and metadata and for adding annotations, CMCS provides an electronic laboratory notebook application that can be launched from its portal and stores its output directly in the CMCS repository. The CMCS portal allows researchers to access heterogeneous data and directly integrate community data, tools, and processes. The infrastructure provides the multiple information pathways necessary to integrate tools and allow researchers to maintain a focus on their science rather than the mechanics of data transfer. Community data exchange (including translation and extraction mechanisms) and standard programming interfaces are designed into the portal, underlying services, and the data/metadata repository to make it simple to integrate thirdparty data and application resources without requiring modifications that would prohibit these resources from continued independent development.

These are all issues that will present challenges to knowledge grids spanning virtual organizations such as a distributed initiative to develop materials for advanced reactors. As knowledge grids lower barriers to discovering, analyzing, and generating physical information, technologies and research processes will need to co-evolve. Researchers will need to participate in multiple communities, and sub-communities will need to be able to independently develop and evolve their domain resources while contributing to multiscale goals.

\subsection{High Watermark Computations in Materials Science and Outlook}

This report acknowledges many challenges in system-level integration of materials science simulations. However, it must also emphasize that the time is right to assume these challenges, in view of the outstanding successes of the materials community in simulating individual systems. We conclude this section by highlighting one area of the computational materials, out of many that were presented at the workshop: molecular dynamics. This highlight is paradigmatic of progress in contemporary materials science simulations in that it pushes the frontier of scientific discovery, it stands at the current end of a long line of algorithmic improvements, it embodies best practices of scientific software engineering, and it runs scalably in the massively parallel environment of hierarchical distributed memory ASCI machines.

Molecular dynamics (MD) is a simulation tool that is useful in solid state, surface physics, chemical physics, high-pressure physics, nanotechnology, and biochemistry. With potentials based on first principles, it is capable of making predictions without inputs from empirical parameters or experiments, for molecules of modest but ever increasing complexity [21,22]. With empirical potentials, molecular dynamics is capable of giving insight into million-atom ensembles up to the nanosecond range, including crack propagation, friction, dislocation dynamics, shock waves, and structural phase transitions [23]. In the context of materials for advanced reactors, it is relevant to vacancy and self- 
interstitial energy calculations, He-vacancy interactions, validation of interatomic potentials, and erosion of the first wall.

The Kohn-Sham density functional approach to molecular dynamics involves coupled nonlinear integro-differential equations whose numerical analysis has complexity $O\left(N^{3}\right)$ for an $N$-electron system. The key kernels are the 3D complex FFT and dense matrix multiplication, both of which are available in highly optimized software. The largest first-principles MD simulation to date appears to be a 1280-atom (3840-electron) high-pressure Boron simulation that consumed two weeks on Lawrence Livermore National Laboratory's MCR Linux cluster with 2000 CPUs, using the code Qbox, implemented with MPI. Such simulations are yielding insight in the complex interplay between interstitial disorder and electronic structure, and follow recent experiments suggesting that Boron undergoes an amorphization under pressure. From the perspective of exploitation of advanced computational platforms, the results are equally encouraging as on the scientific front. Excellent strong scaling (92\%) accompanies the last doubling from 980 to $1960 \mathrm{CPUs}$, and excellent per-processor performance is achieved (up to $40 \%$ of peak). Algorithmic improvements are needed to take first-principles MD to the next order of magnitude of $10^{4}$ atoms, where the $O\left(N^{3}\right)$ cost of orthogonalization of the eigenfunctions dominates. The Holy Grail of electronic structure methods is to achieve linear scaling with controlled accuracy. Some of the first applications run on the computer that is expected to become the world's largest as soon as its installation is complete in 2005 , the BlueGene/L machine, will be molecular dynamics, and the problem scales possible on this new platform will strengthen pressure to reduce the asymptotic computational complexity to linear.

The breathtaking advances in molecular dynamics simulations - in many flavors to answer many different types of scientific questions - exemplify the potential of interdisciplinary teams of scientists, mathematicians, and computer scientists working in teams and poking at the interfaces between the disciplines. MD has benefited from many algorithmic breakthroughs and software investments made in the abstract, but it has also driven progressive aspects of computer science, such as the SWIG wrapper generator, which was developed in conjunction with the Los Alamos MD code SpaSM [24]. A simulation program in materials for advanced reactors will stimulate such advances throughout computational science en route to fulfilling its appropriate role in drastically reducing the number of physical experiments that must ultimately be completed.

\section{References}

1. D. E. Post and R. P. Kendall, Lessons Learned From ASCI, International Journal of High Performance Computing Applications, 2004, in press.

2. D. E. Keyes, (editor-in-chief), A Science-based Case for Large-scale Simulation, U.S. Department of Energy, Washington, DC, 2003.

3. J. D. Myers, T. C. Allison, S. Bittner, B. Didier, M. Frenklach, W. H. Green, Jr., Y.-L. Ho, J. Hewson, W. Koegler, C. Lansing, D. Leahy, M. Lee, R. McCoy, M. Minkoff, S. Nijsure, G. von Laszewski, D. Montoya, C. Pancerella, R. Pinzon, W. Pitz, L. A. Rahn, B. Ruscic, K. Schuchardt, E. Stephan, A. Wagner, T. Windus, and C. Yang, A Collaborative informatics Infrastructure for Multiscale Science, 2004, Proceedings of the Challenges of Large Applications in Distributed Environments (CLADE) Workshop (June 7, 2004, Honolulu).

4. L. Christodoulou, Accelerated Insertion of Materials, http://www.darpa.mil/dso/thrust/matdev/aim, 2003.

5. A. Brandt, in Multiscale and Multiresolution Methods, T. Barth, et al., Eds., Springer, New York, 2001, pp. 3-95.

6. L. Greengard, and V. Rokhlin, A Fast Algorithm for Particle Simulations, J. Comp. Phys. 73 (1987) 325-348.

7. M. H. Kalos, and P. A. Whitlock, Monte Carlo Methods, John Wiley \& Sons, New York, 1986, p. 186. 
8. J. F. Thompson, B. K. Soni, and N. Weatherill, Handbook of Grid Generation, CRC Press, Boca Raton, 1998.

9. L. S. Blackford, J. Choi, A. Cleary, E. D'Azevedo, J. Demmel, I. Dhillon, J. Dongarra, S. Hammarling, G. Henry, A Petitet, K. Stanley, D. Walker and R. C. Whalley, ScaLAPACK Users' Guide, SIAM, Philadelphia, 1997.

10. R. Barrett, M. Berry, T. F. Chan, J. Demmel, J. Dunato, J. Dongarra, V. Eijkhout, R. Pozo, C. Romine and H. van der Vorst, Templates for the Solution of Linear Systems: Building Blocks for Iterative Methods, SIAM, Philadelphia, 1997.

11. U. M. Ascher, and L. R. Petzold, Computer Methods for Ordinary Differential Equations and Differential-Algebraic Equations, SIAM, Philadelphia, 1998.

12. J. Nocedal and S. J. Wright, Numerical Optimization, Springer, New York, 1999.

13. Z. Bai,, J. Demmel, J. Dongarra, A. Ruhe and H. van der Vorst, Templates for the Solution of Algebraic Eigenvalue Problems, SIAM, Philadelphia, 2000.

14. T. DeMarco, The Deadline, Dorset House Publishing, New York, 1997, 310.

15. D. Phillips, The Software Project Manager's Handbook, IEEE Computer Society, Los Alamitos, 1997.

16. R. Thomsett, Radical Project Management, Prentice Hall, Upper Saddle River, NJ, 2002.

17. P. J. Roache, Verification and Validation in Computational Science and Engineering, Hermosa Publishers, Albuquerque, 1998, 446.

18. W. Oberkampf, and T. Trucano, Verification and Validation in Computational Fluid Mechanics, Progress in Aerospace Studies 38 (2002) 209-272.

19. P. J. Roache, Code Verification by the Method of Manufactured Solutions, Transactions of the ASME 124 (2002) 4-10.

20. D. E. Post and R. P. Kendall, Lessons Learned From ASCI, in DOE Software Quality Forum 2003, Los Alamos National Laboratory, Washington, DC, 2003.

21. J.-L. Fattebert and F. Gygi, J. Comput. Chem. 23 (2002) 662-666.

22. T. Ogitsu, E. Schwegler, F. Gygi and G. Galli, Phys Rev. Let. 91 (2003) 175502.

23. K. Kadau, T. C. Germann, P. S. Lomdahl and B. L. Holian, Science, 296 (2002) 1681-1684.

24. P. S. Lomdahl, and D. M. Beazley. Multi-Million Particle Molecular Dynamics on MPPs, in Lecture Notes in Computer Science 1041 (1995) 391-407. 


\section{Summary and Recommendations}

The consensus recommendations of the workshop participants are: (1) a more extensive program of theory and modeling could make significant progress in dealing with critical issues related to structural materials application in Gen-IV and fusion reactors during the next 5 to 10 years, and (2) experiments in prototypic irradiation facilities such as materials test (fission) reactors and $14 \mathrm{MeV}$ neutron sources will be required for validation of model predictions and to support design of the advanced nuclear energy systems. The continuing advances in computational science and computer hardware make this a good time to initiate the new effort in computational materials science. The basis for these recommendations is summarized below.

The overarching goal of the envisioned modeling program is to develop a robust understanding of key physics properties that must simultaneously span length and time scales ranging from atomistic to the continuum and sub-picosecond to years. In contrast with many other problems in materials science, radiation materials science essentially never deals with an equilibrium situation. The system of interest is continually being driven from equilibrium such that events occurring on the smallest time and length scales remain important even at long times. A prioritized list of objectives for the coming decade would include the following tasks to accomplish this goal:

- Electronic structure calculations to obtain intrinsic and defect properties in iron and its alloys, including the effects of $\mathrm{He}$ and $\mathrm{H}$ (this may require advances in theory beyond the current density functional theory).

- Development of computationally efficient and physically robust interatomic alloy potentials, including directional bonding and magnetism (to accurately describe complex multi-component, multiphase materials).

- Advanced large-scale, atomistic models that describe the numerous material parameters and processes that interact in complex ways to control the migration, interaction, and accumulation of defects and gases, as well as the non-equilibrium rearrangements of solute constituents by segregation and phase transitions (to predict nano-scale evolutions in complex materials for both processing and extended service).

- Linked and multiscale (atomistic, mesoscopic, and continuum) deformation and fracture models for predicting hardening, plastic instabilities, changes in ductile-tobrittle fracture, dimensional instabilities, and creep/creep rupture behavior for complex materials and loading conditions.

Because of the nature of the radiation damage problem, the proposed multiscale modeling effort involves using a broad range of computational models rather than attempting to develop a single monolithic model. The appropriate tools and methods should be applied to each scale or aspect of the problem. For example, large scale molecular dynamics simulations of dislocation dynamics and dislocation-defect interactions will be enabled by the next generation of computers. These simulations will provide understanding of deformation and fracture phenomena such as crack nucleation and dislocation channeling with its concomitant flow localization. This insight can be embedded in improved models for rule-based, large-scale 3D dislocation dynamics and constitutive relationships in finite element models that can be used for design. The modeling activities should also apply the lessons learned from the successes and failures of the ASCI enterprise.

The question of determining an adequate level of resources for the required modeling effort may at least partially be addressed by reference to the ASCI program within the DOE's stockpile stewardship program. The level of complexity and the requirement for robust physical models able to describe the properties and performance of a wide array of materials under extreme conditions are similar for both the advanced nuclear energy materials programs and the stockpile stewardship program. Many of the underlying physical phenomena are also common to the materials of interest to fusion and fission 
reactors and stockpile stewardship. For example, production of atomic displacements and helium are primary issues in both cases. The defects are produced in nuclear weapons when the transuranic elements decay by alpha particle emission, rather than by neutron bombardment and nuclear transmutation reactions. Of course, the nature of some of the materials issues are different, notably plutonium's uniquely complex metallurgical behavior. But the level of complexity inherent in the reactor materials arena is also very high. A single fusion (or fission) reactor involves multiple materials being irradiated over a range of elevated temperatures, at high and varying dose and helium production rates, in very complex geometries and loading conditions, and in contact with potentially corrosive coolants. In addition, multiple candidate materials exist and must be evaluated for each application.

In contrast to their relative complexities (but reflecting the importance of national defense), the level of effort for materials modeling alone within the ASCI program is roughly a factor of 3 times larger than the combined experimental and theoretical efforts within the current US fusion materials program. Clearly, more resources are required for theory and modeling to make significant progress on the major scientific issues related to fusion and advanced fission reactor materials if such efforts are expected to reduce the scope of experimental work that will be needed to generate the data required to support reactor design.

It was also pointed out that the desired objectives of an expanded modeling effort will not be achieved simply by increasing access to high speed computing or by building faster computers. In addition to high speed computer cycles, progress is needed in the software arena (such as improved parallel algorithms and methods), and in what might be called the man-machine interface (more standardized interfaces to specialized software packages and improved visualization). These needs can only be addressed by more support for personnel. Similarly, on the materials science side, the advances required to overcome the current challenges to obtaining a successful predictive capability for the DT fusion environment will not be achieved without significant breakthroughs in physical insight into the response of materials to irradiation, and the incorporation of that insight into theoretical models. The potential for advanced models to reveal new mechanisms and outcomes needs to be exploited to prevent technical surprises from short-circuiting the promise of fusion. Materials development is almost certainly the rate-limiting step in the development of fusion as an energy source, and the necessary progress will not be made in a timely way without increasing the level of personnel support. One rule of thumb discussed at the workshop was that progress in a computationally-intensive science enterprise requires balanced financial support for three components: computer hardware, computer operations, and researcher time.

In order for a modeling effort focused on understanding and predicting the effects of radiation on materials to be successful, it must be closely integrated with a complementary experimental activity. This experimental component must ultimately include two types of experiments:

(1) Studies of critical phenomena and mechanisms as required for model development and validation. This will involve carefully controlled experiments on both model materials and engineering alloys in order to elucidate the effects of key material and irradiation variables, and

(2) Engineering experiments that will ultimately confirm the validity of the modeling and simulation results, help develop the database for alloy selection and optimization, and for engineering design.

The fundamental experiments are needed to provide measured values for currently-unknown material parameters required as input to the models, and to verify model predictions by comparison. Depending on the specific issue or parameter of interest, the fundamental experiments may include charged particle (electron, light and heavy ion) irradiations as well as irradiations in materials test reactors or $14 \mathrm{MeV}$ neutron sources. For example, ion irradiation facilities funded by the Office of Science have previously been extensively used to investigate the effects of helium and hydrogen in irradiated materials. These experiments involved dual and triple ion beam facilities in which a heavy ion 
beam (e.g. $\mathrm{Ni}$ or $\mathrm{Fe}$ ) was used to produce displacement damage while $\mathrm{He}$ and $\mathrm{H}$ were simultaneously implanted at the desired rate using the additional beam lines. Such experiments are not fully predictive of neutron irradiation conditions because of issues associated with the limited irradiation volume (typical damage zones are only 1 to 2 micron thick), but they provided considerable insight into the mechanisms by which transmutant gases can influence microstructural evolution (e.g. see the discussion in 3.3.2).

Irradiation at spallation neutron sources also provides an opportunity to investigate helium and hydrogen production at levels even higher than under DT fusion conditions. For example, the spallation neutron sources at the Los Alamos National Laboratory and the Paul Scherrer Institute in Switzerland have been used for this purpose. The possibility of adding a materials irradiation test stand at the SNS has also been proposed. Although some fundamental materials behavior issues can be investigated in these facilities, they are limited to low doses (e.g. $\sim 3 \mathrm{dpa} /$ year in one proposed SNS facility option) compared to the doses at which design data is needed (>100 dpa). Moreover, differences between fusion and spallation neutron spectra at very high energies may give rise to unanticipated effects, e.g. due to the quite different solid transmutation products.

Comparison of model predictions with results from fundamental experiments will provide a feedback mechanism for model improvement, which may lead to the design of additional special purpose experiments. Successful predictions of data from the engineering experiments will provide the confidence needed to use the models to support reactor design. This is the most challenging task for the modeling effort because a true predictive ability is necessary to extrapolate beyond the available database. This also demonstrates the essential need for a broad modeling effort. Just as modeling can not independently solve the materials problems, neither can an experimental program. Given the complexity of the engineering systems, the multitude of materials, and the range of exposure conditions (neutron flux, fluence, temperature, and applied load), it is impossible to develop a complete experimental design basis for even a single advanced fission or fusion reactor design.

Because a successful modeling effort must have a strong microstructural foundation, the development and application of high-resolution materials characterization techniques, such as atom probe field-ion microscopy, electron microscopy, and neutron and X-ray scattering, are very important. Significant advances in materials characterization techniques have been achieved in recent years. Capabilities and facilities required to fully exploit these techniques for irradiated materials are critical to the success of the theory and modeling efforts. This particularly includes maintaining adequate hot cell capacity in the national laboratory system for handling radioactive materials for subsequent testing and examination.

In spite of the potential for computational materials science to prove useful in designing materials and predicting materials' performance, the current reach of the science is limited. Theory and modeling can be used to develop understanding of known critical physical phenomena, and computer experiments can, and have been used to, identify new phenomena and mechanisms, and to aid in alloy design. However, it is questionable whether the science will be sufficiently mature in the foreseeable future to provide a rigorous scientific basis for predicting critical materials' properties, or for extrapolating well beyond the available validation database. This conclusion rests firmly on two very practical considerations: the need for reactor licensing and capital investment.

Even a high degree of scientific confidence that a given alloy will perform as needed in a particular Gen-IV or fusion environment is not necessarily transferable to the reactor licensing or capital market regimes. The philosophy, codes, and standards employed for reactor licensing are properly conservative with respect to design data requirements. Experience with the U.S. Nuclear Regulatory Commission indicates that only modeling results that are strongly supported by relevant, prototypical data will have an impact on the licensing process. In a similar way, it is expected that investment on the scale required to build a fusion power plant (several billion dollars) could only be obtained if a very 
high level of confidence exists that the plant will operate long and safely enough to return the investment.

These two concerns appear to dictate that an experimental facility capable of generating a sufficient, if limited, body of design data under essentially prototypic conditions (i.e. with $\sim 14 \mathrm{MeV}$ neutrons) will ultimately be required for the commercialization of fusion power. An aggressive theory and modeling effort will reduce the time and experimental investment required to develop the advanced materials that can perform in a DT fusion reactor environment. For example, the quantity of design data may be reduced to that required to confirm model predictions for key materials at critical exposure conditions. This will include some data at a substantial fraction of the anticipated end-of-life dose, which raises the issue of when such an experimental facility is required. Long lead times for construction of complex facilities, coupled with several years irradiation to reach the highest doses, imply that the decision to build any fusion-relevant irradiation facility must be made on the order of 10 years before the design data is needed.

Finally, two related areas of research can be used as reference points for the expressed need to obtain experimental validation of model predictions. Among the lessons learned from ASCI, the importance of code validation and verification was emphasized at the workshop. Despite an extensive investment in theory and modeling of the relevant physics, the NIF is being built at LLNL to verify the performance of the physics codes. Similarly, while the U.S. and international fusion community has invested considerable resources in simulating the behavior of magnetically-confined plasmas, a series of experimental devices (e.g. DIII-D, TFTR, JET, NSTX, and NCSX) have been, or will be, built and numerous experiments carried out to validate the predicted plasma performance on the route to ITER and a demonstration fusion power reactor.

In closing, it is important to emphasize that additional resources, and even a dedicated test facility, in and of themselves, will not be enough ensure the successful implementation of advanced nuclear technologies. In reality, the feasibility of fusion and many Gen-IV concepts as large scale energy sources will hinge on the development of new and unique materials. Indeed, success in this effort cannot be guaranteed. Developing such materials will require new ideas and a range of new cutting edge science. The science must be closely coupled with and directed at the unique engineering challenges posed by fusion and advanced fission power. An apt analogy is the support that basic biological sciences provides to modern medicine. The need to develop new, non- $\mathrm{CO}_{2}$ producing energy sources that can serve mankind for generations to come warrants the required level of investment in both theory and modeling, and experimental research facilities. 


\title{
Appendix A Workshop Program and Agenda ${ }^{1}$
}

\section{Workshop on Advanced Computational Materials Science: Application to Fusion and Generation IV Fission Reactors}

\author{
Sponsored by: \\ U.S. Department of Energy \\ Office of Science \\ Office of Nuclear Energy, Science, and Technology
Loews L'Enfant Plaza Hotel
Washington, DC
March 31-April 2, 2004 \\ Workshop Organizers \\ Bill Corwin, Oak Ridge National Laboratory \\ Jeff Nichols, Oak Ridge National Laboratory \\ Roger Stoller, Oak Ridge National Laboratory \\ Steve Zinkle, Oak Ridge National Laboratory \\ International Advisory Committee \\ Patrick Ledermann, Commissariat à l'énergie atomique (CEA), France \\ David Bacon, University of Liverpool, United Kingdom \\ Nasr Ghoniem, UCLA, USA \\ David Keyes, Columbia University, USA \\ Tetsuya Sato, Earth Simulator Center, Japan \\ $\underline{\text { Administrative Staff }}$ \\ Christie Ashton, U.S. Department of Energy \\ Pam Hadley, Oak Ridge National Laboratory
}

\footnotetext{
${ }^{1}$ Note: A complete copy of workshop presentations can be found on the following website: http://www.csm.ornl.gov/meetings/SCNEworkshop/DC-index.html, or obtained from one of the ORNL Organizers listed above.
} 


\section{Program Overview}

\begin{tabular}{|c|c|c|}
\hline $\begin{array}{l}\text { Wednesday, } 31 \text { March } \\
\text { (Panelists meet at 7:45 am) }\end{array}$ & Thursday, 1 April & Friday, 2 April \\
\hline $\begin{array}{c}8: 30-9: 00 \text { am } \\
\text { DOE SC-NE Welcome and } \\
\text { Charge to Panel }\end{array}$ & & \\
\hline $\begin{array}{c}\text { 9:00-10:10 am } \\
\text { Session I(a) }\end{array}$ & $\begin{array}{l}\text { 8:30-10:00 am } \\
\underline{\text { Session III(a) }}\end{array}$ & $8: 30-12: 00$ noon \\
\hline 10:10 - 10:30 am Break & 10:00 - 10:20 am Break & Panelists and Organizers \\
\hline $\begin{array}{c}10: 30-11: 30 \mathrm{am} \\
\text { Session } \mathbf{I}(\mathbf{b})\end{array}$ & $\begin{array}{c}10: 20-11: 45 \mathrm{am} \\
\underline{\text { Session III(b) }}\end{array}$ & $\begin{array}{l}\text { Breakout sessions and document } \\
\text { preparation }\end{array}$ \\
\hline 11:30 - 12:45 Lunch & 11:45 - 1:00 pm Lunch & 12:00 - 1:00 Wrap-up Lunch \\
\hline $\begin{array}{c}12: 45-2: 30 \mathrm{pm} \\
\text { Session II(a) }\end{array}$ & $\begin{array}{c}\text { 13:00-2:30 pm } \\
\underline{\text { Session IV(a) }}\end{array}$ & \\
\hline 2:30 - 2:50 pm Break & 2:30 - 2:50 pm Break & \\
\hline \multirow{2}{*}{$2: 50-5: 45 \mathrm{pm}$} & $\begin{array}{l}2: 50-4: 00 \mathrm{pm} \\
\text { Session IV(b) }\end{array}$ & \\
\hline & $\begin{array}{c}\text { 4:00-5:00 pm } \\
\text { Session V(a) } \\
\text { Panelists and Organizers }\end{array}$ & \\
\hline $\begin{array}{c}\quad 6: 45 \mathrm{pm} \\
\text { Open reception: All }\end{array}$ & $\begin{array}{c}6: 00-9: 30 \mathrm{pm} \\
\text { Working dinner: Panelists and } \\
\text { Organizers } \\
\text { Session } \mathrm{V}(\mathrm{b})\end{array}$ & \\
\hline
\end{tabular}




\section{DOE SC-NE Welcome and Charge to Panel - Chairman James Roberto (ORNL)}

William D. Magwood, IV, Director, DOE Office of Nuclear Energy

Raymond L. Orbach, Director, DOE Office of Science

Roger E. Stoller, Co-organizer, Oak Ridge National Laboratory

Session I: Overview of relevant irradiation environments (Charge-d) $-\underline{2 \text { hours }}$

\section{Program}

Bill Wolfer, introduction to radiation damage in materials

Todd Allen, Gen-IV fission reactor environments

Steve Zinkle, fusion and spallation irradiation conditions

Jean-Louis Boutard, EU programs on simulating radiation damage environments

\section{Cognizant Subpanel}

\begin{tabular}{|l|l|}
\hline $\begin{array}{l}\text { Patrick Ledermann, Commissariat à l'Energie Atomique (CEA), } \\
\text { France }\end{array}$ & $\begin{array}{l}\text { Session Chairman, International } \\
\text { Advisory Committee }\end{array}$ \\
\hline Todd Allen, University of Wisconsin & \\
\hline Jean-Louis Boutard, Commissariat à l'Energie Atomique & \\
\hline Wilhelm G. Wolfer, Lawrence Livermore National Laboratory & \\
\hline Bill Corwin, Oak Ridge National Laboratory & Organizer (NE) \\
\hline Steve Zinkle, Oak Ridge National Laboratory & Organizer (OFES) \\
\hline
\end{tabular}


Session II: Current state of the art in relevant multiscale computational materials science models. What phenomena can be adequately modeled? (Charge-b) $-\underline{4.5 \text { hours }}$

\section{Program}

Malcolm Stocks, $a b$ initio methods

Michael Moseler, interatomic potentials

Art Voter (with Robert Averback and Stephen Foiles) atomistic simulations, MD

Michel Guttmann, kinetic methods, MC, multiscale modeling

Tomas Diaz de la Rubia, insights from ASCI research, dislocation dynamics

Greg Olson (with Dennis Dimiduk), integrated methods in materials design, AIM project

Hideo Kaburaki, MD study on radiation hardening and fracture processes in FCC metals

\section{Cognizant Subpanel}

\begin{tabular}{|l|l|}
\hline David Bacon, University of Liverpool, United Kingdom & $\begin{array}{l}\text { Session Chairman, International } \\
\text { Advisory Committee }\end{array}$ \\
\hline Graeme Ackland, University of Edinburgh, United Kingdom & \\
\hline Robert Averback, University of Illinois & \\
\hline Stephen Foiles, Sandia National Laboratory & \\
\hline $\begin{array}{l}\text { Michael Moseler, Fraunhofer Institute for Mechanics of Materials, } \\
\text { Germany }\end{array}$ & \\
\hline $\begin{array}{l}\text { Michel Guttmann, Commissariat à l'Energie Atomique (CEA), } \\
\text { France }\end{array}$ & \\
\hline Japan Kaburaki, Japan Atomic Energy Research Institute (JAERI), & \\
\hline Greg Olson, Northwestern University & Organizer (OBES) \\
\hline Malcolm Stocks, Oak Ridge National Laboratory & \\
\hline Valentin Vaks, Kurchatov Institute, Russia & \\
\hline Art Voter, Los Alamos National Laboratory & \\
\hline Roger Stoller, Oak Ridge National Laboratory & \\
\hline
\end{tabular}


Session III: Where do we need to go? Goals and targets for understanding and predicting material performance (Charge-a) - $\underline{3 \text { hours }}$

\section{Program}

Bob Odette, deformation and fracture mechanisms and related phenomena Roger Stoller (with Todd Allen), helium effects on void swelling and IASCC David Bacon, dislocation interactions, dislocation dynamics Georges Martin, open questions in modeling behavior of concentrated alloys Graeme Ackland, potential future for improved and alloy interatomic potentials Malcolm Stocks, relevant future directions in $a b$ initio, and linking to other methods

\section{Cognizant Subpanel}

\begin{tabular}{|l|l|}
\hline Nasr Ghoniem, UCLA & $\begin{array}{l}\text { Session Chairman, International } \\
\text { Advisory Committee }\end{array}$ \\
\hline Graeme Ackland, University of Edinburgh, United Kingdom & \\
\hline Todd Allen, University of Wisconsin & \\
\hline $\begin{array}{l}\text { Tomas Diaz de la Rubia, Lawrence Livermore National } \\
\text { Laboratory }\end{array}$ & \\
\hline Sergei Dudarev, UK AEA, Culham, United Kingdom & \\
\hline $\begin{array}{l}\text { Georges Martin, Commissariat à l'Energie Atomique (CEA), } \\
\text { France }\end{array}$ & \\
\hline Jean Claude Van Duysen, Electricité de France (EDF), France & Organizer (OBES) \\
\hline G. Robert Odette, University of California, Santa Barbara & \\
\hline Art Voter, Los Alamos National Laboratory & \\
\hline Steve Zinkle, Oak Ridge National Laboratory & \\
\hline Roger Stoller, Oak Ridge National Laboratory & \\
\hline
\end{tabular}


Session IV: What can advanced computational science be expected to provide, i.e. expected progress over next $\sim 10$ years? (Charge-c) $-\underline{2.5 \text { hours }}$

\section{Program}

Bill Gropp, Hardware and software environments for high-end simulation

Doug Post, Lessons learned from ASCI software projects

Francois Gygi, Current limits of first principles simulations

Leslie Greengard, Fast algorithms for potential problems and molecular dynamics

David Keyes, Lessons learned from SciDAC and software from the SciDAC ISICs

\section{Cognizant Subpanel}

\begin{tabular}{|l|l|}
\hline David Keyes, Columbia University & $\begin{array}{l}\text { Session Chairman, International } \\
\text { Advisory Committee }\end{array}$ \\
\hline Leslie Greengard, New York University & \\
\hline Douglass E. Post, Los Alamos National Laboratory & \\
\hline Francois Gygi, Lawrence Livermore National Laboratory & \\
\hline Rick Stevens, Argonne National Laboratory & \\
\hline Malcolm Stocks, Oak Ridge National Laboratory & \\
\hline Jeff Nichols, Oak Ridge National Laboratory & Organizer (ASCR) \\
\hline
\end{tabular}


Session V: $\quad$ How can we bridge the gap between current knowledge and capabilities and the defined

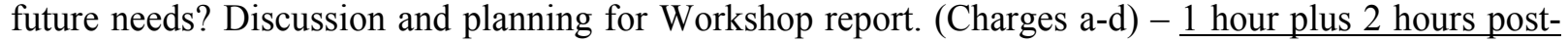
dinner discussion

\section{Program}

Doug Post, Perspectives on Materials Computational Science

Colin English, Discussion Moderator

Overall discussion of workshop goals and presentations, e.g.

a. expected contribution of computational methods and development

b. role of ongoing experimental activities, possible environments for radiation damage simulation appropriate to the desired application

c. relationship and balance between theory/modeling and experiments, e.g. use of modeling to suggest critical experiments and role of experimental data in model verification

\section{Cognizant Subpanel}

\begin{tabular}{|l|l|}
\hline Colin English, British Nuclear Fuels, plc., United Kingdom & Session Chairman \\
\hline Tetsuya Sato, Earth Simulator Center, JAPAN & $\begin{array}{l}\text { International Advisory } \\
\text { Committee }\end{array}$ \\
\hline Dennis Dimiduk, Air Force Research Laboratory, WPAFB & \\
\hline Hans Ludewig, Brookhaven National Laboratory & \\
\hline Hideki Matsui, Tohoku University, Japan & \\
\hline Douglass E. Post, Los Alamos National Laboratory & \\
\hline
\end{tabular}




\section{Appendix B Workshop Participants}

$\underline{\text { Organizers from Oak Ridge National Laboratory }}$

William R. Corwin, Metals and Ceramics Division

Jeffrey A. Nichols, Computer Science and Mathematics Division

corwinwr@ornl.gov

Roger E. Stoller, Metals and Ceramics Division

Steven J. Zinkle, Metals and Ceramics Division

nicholsja@ornl.gov

stollerre@ornl.gov

zinklesj@ornl.gov

International Advisory Committee

David Bacon

Nasr Ghoniem

David Keyes

Patrick Ledermann

Tetsuya Sato

$\underline{\text { Invited Panelists }}$

Graeme Ackland

Todd Allen

Bob Averback

Jean-Louis Boutard

Tomas Diaz de la Rubia

Dennis Dimiduk

Sergei Dudarev

Colin English

Stephen Foiles

Leslie Greengard

Michel Guttmann

Francois Gygi

Hideo Kaburaki

Hans Ludewig

Michael Moseler

Georges Martin

Hideki Matsui

Bob Odette

Greg Olson

Douglass Post

Rick Stevens

Malcolm Stocks

Marshall Stoneham

Valentin Vaks

Jean-Claude Van-Duysen

Art Voter

Bill Wolfer

\section{$\underline{\text { Observers }}$}

John Aidun

Charles Baker

Sam Berk

Roberto Car

Alfredo Caro djbacon@liverpool.ac.uk

ghoniem@ucla.edu

david.keyes@columbia.edu

ledermann@aquilon.cea.fr

tetsuya@jamstec.go.jp

g.j.ackland@edinburgh.ac.uk

allen@engr.wisc.edu

averback@uiuc.edu

jean-louis.boutard@cea.fr

delarubia@1lnl.gov

dennis.dimiduk@wpafb.af.mil

sergei.dudarev@ukaea.org.uk

english.colin@nuclear-sciences.co.uk

foiles@sandia.gov

greengar@cims.nyu.edu

michel.guttmann@cea.fr

fgygi@1lnl.gov

kaburaki@sugar.tokai.jaeri.go.jp

ludewig@bnl.gov

moseler@iwm.fraunhofer.de

gpmartin@cea.fr

matsui@imr.edu

odette@engineering.ucsb.edu

g-olson@nwu.edu

post@lanl.gov

stevens@mcs.anl.gov

stocksgm@email.cind.ornl.gov

a.stoneham@ucl.ac.uk

vaks@mbslab.kiae.ru

jean-claude.van-duysen@edf.fr

afv@lanl.gov

wolfer1@1lnl.gov

aidun@sandia.gov

cbaker@it.ucsd.edu

sam.berk@science.doe.gov

rcar@princeton.edu

caro2@1lnl.gov 


Yok Chen
Stephen Dean
Anter El-Azab
Huei Fang
Helen Farrell
Jian Gan
F. Xabier Garaizar
Dolores Gomez-Briceno
Robert Gottschall
Diane Graziano
Lance Greenwade
John Herczeg
Michael James
Stephanie Jumel
Tina Kaarsberg
Alison Kubota
Richard Kurtz
Susan Lesica
Christian Mailhiot
Louis Mansur
Stanley Milora
J. Manuel Perlado
Jeffrey Rest
James Roberto
Kenneth Roche
Salvador Rodriguez
Roman Samulyak
Richard Sheffield
Edward Simonen
Michael Strayer
Clinton Van Siclen
Max Victoria
William Weber
Brian Wirth

Yok Chen

Stephen Dean

Huei Fang

Helen Farrell

Jian Gan

F. Xabier Garaizar

Dolores Gomez-Briceno

Robert Gottschall

Diane Graziano

Lance Greenwade

John Herczeg

Michael James

Stephanie Jume

Tina Kaarsberg

Alison Kubota

Richard Kurtz

Louis Mansur

Jeffrey Rest

James Roberto

Kenneth Roche

Salvador Rodriguez

Roman Samulyak

Richard Sheffield

Edward Simonen

Michael Strayer

Max Victoria

Brian Wirth yok.chen@science.doe.gov

fusionpwrassoc@aolcom

anter.el-azab@pnl.gov

hefang@sandia.gov

farrhh@inel.gov

jian.gan@anl.gov

xabier.garaizar@nnsa.doe.gov

lola.gomezbriceno@ciemat.es

robert.gottschall@science.doe.gov

graziano@cmt.anl.gov

leg@inel.gov

john.herczeg@hq.doe.gov

mrjames@swcp.com

stephanie.jumel@edf.fr

tna.kaarsberg@mail.house.gov

kubota1@1lnl.gov

rj.kurtz@pnl.gov

sue.lesica@hq.doe.gov

mailhoit1@1lnl.gov

mansurlk@ornl.gov

miloras1@ornl.gov

mperlado@din.upm.es

jrest@anl.gov

robertojb@ornl.gov

rochekj@ornl.gov

sbrodri@sandia.gov

rosamu@bnl.gov

sheff@lanl.gov

ed.simonen@pnl.gov

michael.strayer@science.doe.gov

cvs@inel.gov

max.victoria@psi.ch

bill.weber@pnl.gov

bdwirth@nuc.berkeley.edu 


\section{Appendix C Acronyms and Abbreviations}

BES U.S. DOE Office of Basic Energy Sciences, a component of SC

BWR boiling water reactor, an LWR in which the primary coolant water is permitted to boil within the reactor pressure vessel, eliminating the need for external steam generators

dpa displacements per atom, neutron exposure unit that accounts for total kinetic energy absorbed by atoms in a material irradiated by energetic particles

DOE Department of Energy

DT designation for the fusion reaction involving the heavy hydrogen isotopes deuterium and tritium with a 2.X MeV alpha particle (helium atom nucleus) and a $14.1 \mathrm{MeV}$ neutron as the reaction products

GFR gas fast reactor; a He-cooled, fast-spectrum Gen-IV design

Gen-IV Generation-IV, advanced nuclear reactor designs that meet specified criteria for economy, safety, reliability, and proliferation resistance

$\mathrm{He} / \mathrm{dpa}$ ratio of helium production to displacement damage production, parameter used to characterize material exposure in a given irradiation environment, normally expressed in appm $\mathrm{He} / \mathrm{dpa}$

IFMIF International Fusion Materials Irradiation Facility, a proposed accelerator-based neutron source employing a deuteron beam and a lithium target to produce a sufficiently high flux of $14.1 \mathrm{MeV}$ neutrons for fusion materials irradiation and evaluation

ITER International Thermonuclear Experimental Reactor, the burning plasma experiment which is intended to verify DT plasma physics performance for a future demonstration power reactor

LFR lead fast reactor; a lead (or lead alloy)-cooled, fast-spectrum Gen-IV design

LWR light water reactor, current generation of fission reactor with conventional water as coolant

NE U.S. DOE Office of Nuclear Energy. Science, and Technology

NGNP next generation nuclear plant; He-cooled Gen-IV design, an advanced VHTR designed for production of hydrogen and electricity

NRC U.S. Nuclear Regulatory Commission, agency responsible for regulation and licensing of nuclear facilities

NRT an international standard by Norgett, Robinson and Torrens for computing the number of displacements generated by a single PKA

OASCR U.S. DOE Office of Advanced Scientific and Computational Research, a component of SC

OFES U.S. DOE Office of Fusion Energy Sciences, a component of SC

PKA primary knock-on atom, the atom receiving kinetic energy from a collision with an energetic neutron 
PWR pressurized water reactor; an LWR in which the primary coolant water is highly pressurized to prevent boiling, steam is produced in a secondary system steam generator

SC U.S. DOE Office of Science

SCWR supercritical water reactor; a Gen-IV design cooled by supercritical water

SFR sodium fast reactor; a sodium-cooled, fast-spectrum Gen-IV design

VHTR very high temperature reactor; He-cooled, thermal spectrum Gen-IV reactor design operating at high temperature 